\title{
Photoreactivity of donor-acceptor stilbene complexes in a macrocyclic host
}

Christopher T. Eckdahl ${ }^{a}$, Carrie Ou ${ }^{a}$, Suyog Padgaonkara ${ }^{a}$, Mark C. Hersam ${ }^{\mathrm{a}, \mathrm{b}}$, Emily A. Weiss ${ }^{\mathrm{a}, \mathrm{b}}$, Julia A. Kalow ${ }^{*}$

${ }^{a}$ Department of Chemistry, Northwestern University, Evanston, IL 60208, United States

${ }^{b}$ Department of Materials Science and Engineering, Northwestern University, Evanston, IL 60208, United States

*Corresponding author e-mail: j-kalow@northwestern.edu
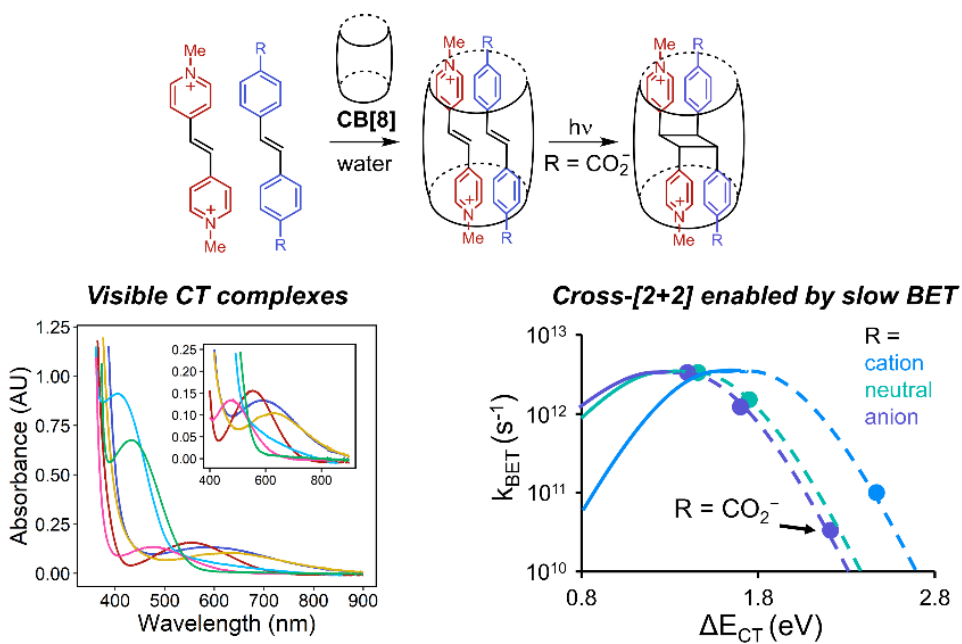

Abstract: Host-guest 2:1 complexation of photoreactive alkene guests improves the selectivity of $[2+2]$ photodimerizations by templating alkene orientation prior to irradiation. Host-guest chemistry can also provide 1:1:1 complexes through the inclusion of electronically complementary donor and acceptor guests, but the photoreactivity of such complexes has not been investigated. We imagined that such complexes could enable selective cross-[2+2] photocycloadditions between donor and acceptor stilbenes. In pursuit of this strategy, we investigated a series of stilbenes and found 1:1:1 complexes with cucurbit[8] uril that exhibited charge-transfer (CT) absorption bands in the visible and near-IR regions. Irradiation of the CT band of an azastilbene, 4,4'-stilbenedicarboxylate, and cucurbit[8] uril ternary complex led to a selective cross-[2+2] photocycloaddition, while other substrate pairs exhibited no chemistry upon CT excitation. Using transient absorption (TA) spectroscopy, we were able to understand the variable photoreactivity of different stilbene donor-acceptor complexes. We found that the back electron transfer (BET) following CT excitation of the photoreactive complex is positioned deep in the Marcus inverted region due to electrostatic stabilization of the ground state, allowing [2+2] to effectively compete with this relaxation pathway. Control reactions revealed that the cucurbit[8] uril host not only serves to template the reaction from the ground state, but also protects the long-lived radical ions formed by CT from side reactions. This protective role of the host suggests that donor-acceptor host-guest ternary complexes could be used to improve existing CT-initiated photochemistry or access new reactivity. 


\section{INTRODUCTION}

The [2+2] photodimerization of alkenes, especially stilbenes, coumarins, and cinnamates, has been extensively studied as a prototypical organic photoreaction. ${ }^{1-11}$ In this reaction, light, typically with wavelengths of $350 \mathrm{~nm}$ or shorter, is used to promote an alkene to the excited stated from which it can engage in a thermally forbidden [2+2] cycloaddition with a second alkene to produce a cyclobutane. Pre-orienting the alkenes for the desired reaction using a macrocylic host molecule, such as $\gamma$-cyclodextrin or cucurbit[8]uril (CB[8]), can improve selectivity for the desired photocycloaddition. ${ }^{1,12-20}$ The guests employed in these templating strategies are often donor-acceptor stilbenes, such as 4-styrylpyridine, which form a head-to-tail 2:1 complex due to favorable $\pi-\pi$ interactions (Figure 1a). Besides improving regio- and diasteroselectivity in small-molecule photochemistry, host-guest complexation can provide photoresponsive supramolecular crosslinks in polymeric systems. ${ }^{12-14,16,21-27}$

Most examples of host-guest-templated [2+2] photocycloadditions begin with the formation of a 2:1 complex followed by photodimerization. ${ }^{1,12-20}$ If a host could pre-arrange two different alkenes in a 1:1:1 ternary complex, then cross-[2+2] chemistry, in which the two reactive stilbenes are different, could be accessed selectively. Previous studies with cinnamates and coumarins have demonstrated size complementarity, in which steric hinderance prevents 2:1 complexation, as an effective means to achieve such 1:1:1 complexation and selectivity for the cross-products (Figure 1b). ${ }^{28-32}$ Electronic donor-acceptor interactions between guests have also been shown to yield 1:1:1 host-guest complexes, but such complexes have not been used to template subsequent photochemistry. ${ }^{33,34}$

We envisioned that donor-acceptor host-guest ternary complexes could be used as templates to achieve selective intermolecular cross-[2+2] stilbene photocycloadditions (Figure 1c). Moreover, such complexes could be excited by visible or even near-infrared light thanks to the emergence of charge transfer (CT) absorption between the donor and acceptor substrates. ${ }^{34,35}$ Typically, CT-initiated photochemistry requires one of the substrates to contain a leaving group because rapid cleavage prevents the initially-formed CT state from quickly relaxing back to the ground state by back electron transfer (BET). ${ }^{36}$ Direct reaction of radical ions formed by CT has rarely been reported due to competition with BET. ${ }^{37-40}$ In fact, most studies of cross-[2+2] chemistry have viewed CT as a nonproductive pathway. ${ }^{3,41-43}$ However, it should be fundamentally possible to directly access [2+2] reactivity from the radical ions produced by $\mathrm{CT}$ absorption, and host-guest complexes offer an ideal platform to investigate such CT-initiated chemistry by providing modular access to well-defined donor-acceptor complexes. ${ }^{34}$ 


\section{Previous Work:}

a

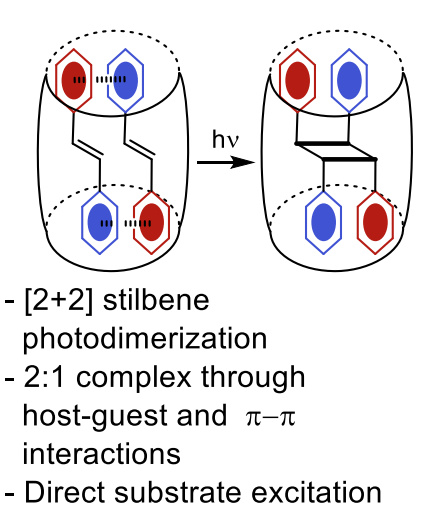

b

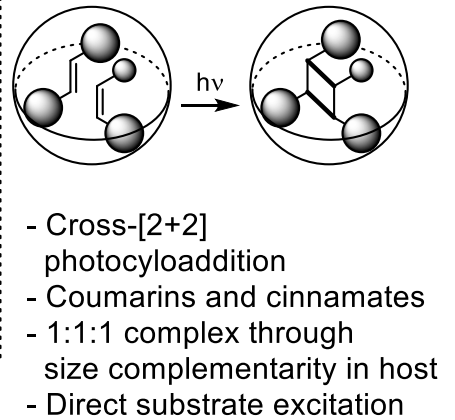

This Work:
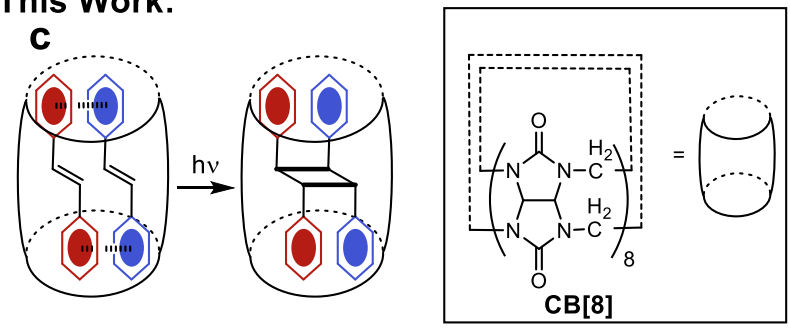

- Cross- [2+2] stilbene photocylcoaddition

$-1: 1: 1$ complex through host-guest and $\pi-\pi$ interactions

- Charge transfer excitation

Figure 1. Previous work has demonstrated (a) head-to-tail [2+2] photodimerizations of donor-acceptor stilbenes templated by host-guest and $\pi-\pi$ interactions. $(\mathrm{red}=$ electron acceptor, blue $=$ electron donor $)$ and (b) sterically controlled host-guest templated cross-[2+2] photocycloadditions of coumarins and cinnamates. (c) In this work, we explore whether electronic donor-acceptor and host-guest interactions can be used to template cross-[2+2] photocycloadditions. Structure of $\mathbf{C B}$ [8] is shown in the bottom right.

In this work, we investigated a series of donor-acceptor host-guest ternary complexes between stilbenes and $\mathbf{C B}[8]$ and discovered that CT reactivity is highly sensitive to the structure of the donor stilbene. While anionic, cationic, and neutral donor stilbenes form 1:1:1 complexes with a dimethylazastilbene acceptor in $\mathbf{C B}[8]$, only one combination led to productive CT-initiated cross[2+2] photocycloaddition. We used ultrafast spectroscopy and computational modeling to show that slow back electron transfer (BET) following CT is necessary for productive [2+2] reactivity. We further found that the cucrbit[8] uril host not only serves to template the ternary complex, but also protects the radical ion intermediates formed by CT, suggesting that host-chemistry could be used to access or improve other CT-initiated reactions. 


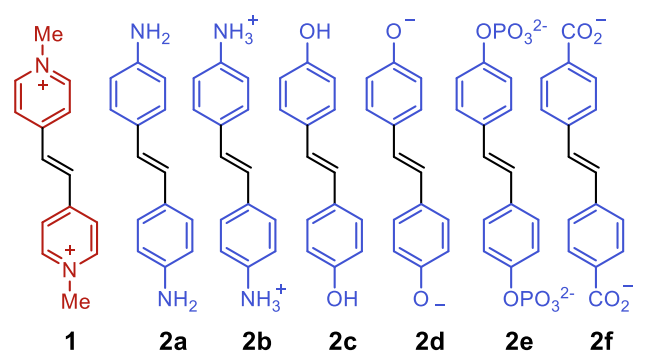

Chart 1. Electron acceptor (1) and donor $(\mathbf{2 a}-\mathbf{2 f})$ stilbenes studied in this work.

\section{RESULTS}

Ramamurthy and coworkers previously showed that protonated 4-styrylpyridines undergo selective UV-mediated photodimerization as 2:1 complexes in $\mathbf{C B}$ [8]. ${ }^{1}$ To enable photochemical studies under both acidic and basic conditions, we chose to investigate an electron-poor dimethylazastilbene dication (1, Chart $\mathbf{1})$ as the electron acceptor. We also selected several parasubstituted stilbenes with variable donating ability and charge as the electron donors (2a-2f, see Experimental Section for synthetic details). We then formed 1:1:1 complexes of the donor, acceptor, and $\mathbf{C B}[8]$ in water (to provide the requisite hydrophobic host-guest interactions) ${ }^{44}$ and characterized these complexes by ${ }^{1} \mathrm{H}$ NMR and UV-Vis spectrophotometry.

To study the formation of 1:1:1 complexes, we added one equivalent of each of the electronrich stilbenes to a $1.5 \mathrm{mM}$ solution of $\mathbf{C B}[8]$ and 1 in neutral, acidic $\left(1 \mathrm{M} \mathrm{D}_{2} \mathrm{SO}_{4}\right)$, or basic $(0.1 \mathrm{M}$ $\left.\mathrm{NaCO}_{3}\right) \mathrm{D}_{2} \mathrm{O}$ (see Experimental Section for details). Acidic or basic solutions were used to protonate or deprotonate the donor stilbenes, respectively; $0.1 \mathrm{M} \mathrm{NaCO}_{3}$ was used because 1 degraded in more basic $1 \mathrm{M} \mathrm{NaOD}$ (likely via single electron reduction by hydroxide, as has been reported for methyl viologen). ${ }^{45}$ The ${ }^{1} \mathrm{H}$ NMR peaks of acceptor 1 shifted upfield upon addition of $\mathbf{C B}$ [8] due to shielding, indicative of host-guest complexation. ${ }^{44}$ The relative integrated areas of bound and free $\mathbf{C B}[\mathbf{8}]$ and $\mathbf{1}$ indicate the formation of a 1:1 complex, 1@CB [8]. Addition of the donor stilbenes leads to further upfield shifts of the signals of $\mathbf{1}$. As an example, Figure $\mathbf{2} \mathbf{b}$ shows the ${ }^{1} \mathrm{H}$ NMR spectrum of $\mathbf{1}$ upon addition of $\mathbf{C B}$ [8] followed by $2 \mathbf{c}$. Relative integrated areas of the signals of 1, 2c, and $\mathbf{C B}$ [8] indicate the formation of a 1:1:1 complex, $1 \cdots 2 \mathbf{c} @ \mathbf{C B}[8]$. ${ }^{1} \mathrm{H}$ NMR spectra for other complexes can be found in SI (Figures S8-S14). We followed the shifts of $\mathbf{1}$ or appearance of new peaks (depending on the timescale of exchange) while titrating $\mathbf{C B}[8]$ and/or donor stilbenes to determine the binding constants for all of the relevant supramolecular interactions (see Figures S15-S22 for titration data). These data are presented in Table 1. During these experiments, we discovered that the phenoxide stilbene, 2d, degraded in ambient conditions over the course of an hour. Some of the ternary complexes formed quantitatively even at the lowest concentrations detectable via ${ }^{1} \mathrm{H}$ NMR, indicating binding constants greater than $10^{5} \mathrm{M}^{-1}{ }^{46}$ Phosphate stilbene $2 \mathrm{e}$ exhibits unique behavior among the donor stilbenes. Addition of $\mathbf{C B}[8]$ to $1 \cdots 2 \mathrm{e}$ leads to downfield shifts of $2 \mathrm{e}$ signals, suggesting that $2 \mathrm{e}$ does not form a ternary complex with $\mathbf{C B}$ [8] and 1, but instead competes with $\mathbf{C B}$ [8] for binding to 1. Unfavorable interactions between the partially negatively charged oxygens of the $\mathbf{C B}$ [8] portal and 
the highly negatively charged phosphate group of $2 \mathrm{e}$ likely prevent its inclusion in a ternary complex (see Figure S12). ${ }^{44}$ The carboxylate-containing stilbene, $\mathbf{2 f}$, weakly binds $\mathbf{1}$ outside of $\mathbf{C B}$ [8], but the host-guest and ternary interactions are at least 1,000 times stronger, such that $>99.9 \%$ of the interactions between $\mathbf{1}$ and $\mathbf{2} \mathbf{f}$ take place within $\mathbf{C B}[\mathbf{8}]$.

\begin{tabular}{|c|c|c|}
\hline Interaction & Condition & Binding Constant $\left(\mathrm{M}^{-1}\right)$ \\
\hline $1 \cdots \mathrm{CB}[8]$ & $\mathrm{D}_{2} \mathrm{O}$ & $(1.3 \pm 0.2) \times 10^{5}$ \\
\hline $2 a \cdots 1 @ C B[8]$ & $\mathrm{D}_{2} \mathrm{O}$ & $>10^{5}$ \\
\hline $2 b \cdots 1 @ C B[8]$ & $1 \mathrm{M} \mathrm{D}_{2} \mathrm{SO}_{4}$ & $(3.8 \pm 0.5) \times 10^{2}$ \\
\hline $2 \mathrm{c} \cdots 1 @ \mathrm{CB}[8]$ & $\mathrm{D}_{2} \mathrm{O}$ & $>10^{5}$ \\
\hline 2f $\cdots 1 @ C B[8]$ & $0.1 \mathrm{M} \mathrm{Na}_{2} \mathrm{CO}_{3}$ in $\mathrm{D}_{2} \mathrm{O}$ & $>10^{5}$ \\
\hline $2 e^{\cdots 1} 1$ & $0.1 \mathrm{M} \mathrm{Na}_{2} \mathrm{CO}_{3}$ in $\mathrm{D}_{2} \mathrm{O}$ & $(4.1 \pm 0.2) \times 10^{2}$ \\
\hline $2 f \cdots 1$ & $0.1 \mathrm{M} \mathrm{Na}_{2} \mathrm{CO}_{3}$ in $\mathrm{D}_{2} \mathrm{O}$ & $(3.37 \pm 0.07) \times 10^{2}$ \\
\hline
\end{tabular}

Table 1. Binding constants of relevant supramolecular interactions. See SI for additional details.

We measured UV-Vis spectra of all the 1:1:1 complexes, in addition to those of the watersoluble stilbenes $(\mathbf{2} \mathbf{a}, \mathbf{2} \mathbf{b}, \mathbf{2} \mathbf{d}, \mathbf{2} \mathbf{e}$, and $\mathbf{2} \mathbf{f})$ on their own. Each of the stilbenes exhibited structured absorption bands in the near-UV with onsets between 300 and $350 \mathrm{~nm}$ (Figure S6). The complexes additionally revealed broad, weak, charge transfer (CT) bands in the visible region (Figure 2c). The 1․2a@CB[8] complex CT band extends well into the near-IR, with an absorption onset of about 900 nm.

a

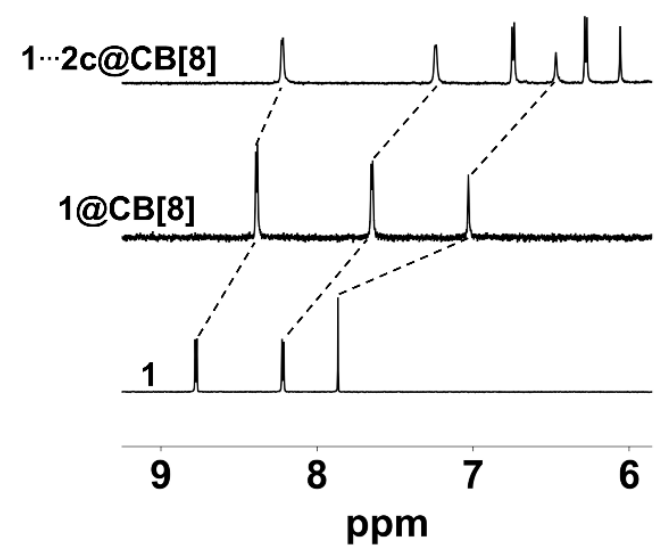

b

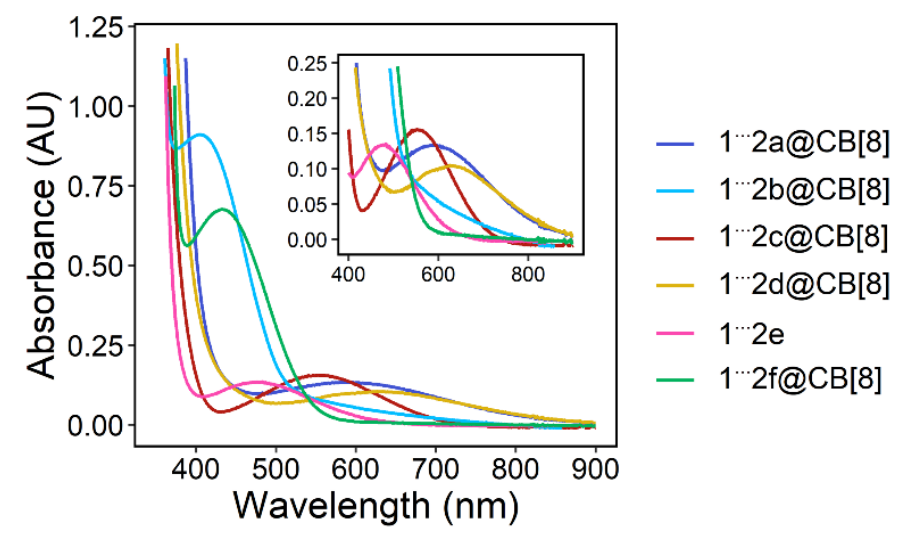

Figure 2. (a) ${ }^{1} \mathrm{H}$ NMR demonstrating supramolecular interactions of $1, \mathbf{C B}[8]$, and $2 \mathrm{c}$. Measured at $1 \mathrm{mM}$ in $\mathrm{D}_{2} \mathrm{O}$. Dotted lines are added to indicate upfield shifts. (b) UV-Vis of charge transfer bands of stilbene donoracceptor complexes (15 $\mathrm{mM}$ with respect to 1$)$. Inset shows the same data with a zoomed-in y axis. Samples containing $\mathbf{2 d}, \mathbf{2 e}$, or $\mathbf{2} \mathbf{f}$ were in $0.1 \mathrm{M} \mathrm{NaCO}_{3}$. Samples containing $\mathbf{2 b}$ were in $1 \mathrm{M} \mathrm{H}_{2} \mathrm{SO}_{4}$. All other samples were in neutral $\mathrm{H}_{2} \mathrm{O}$.

To investigate whether CT could be used to initiate cross-[2+2] reactions, we prepared 1.5 $\mathrm{mM}$ solutions of each complex (the ternary complexes of each pair in $\mathbf{C B}[\mathbf{8}]$, and $\mathbf{1} \cdots \mathbf{2} \mathbf{e}$ without $\mathbf{C B}$ [8]) 
in nitrogen-purged $\mathrm{H}_{2} \mathrm{O}$. We irradiated the charge-transfer bands of each complex using appropriate LEDs: red $(626 \mathrm{~nm})$ for $\mathbf{2 a}$ and $\mathbf{2 d}$, amber $(605 \mathrm{~nm})$ for $\mathbf{2 c}$, green $(525 \mathrm{~nm})$ for $\mathbf{2 e}$, and violet (400 $\mathrm{nm})$ for $\mathbf{2 b}$ and $\mathbf{2 f}$. We analyzed the samples via LCMS following 24 hours of irradiation. The only samples that exhibited conversion were 1 $\cdots \mathbf{2} \mathbf{f} \mathbf{C B}[8]$ and $1 \cdots \mathbf{2} \mathbf{d} @ \mathbf{C B}[\mathbf{8}]$, but the latter complex containing $\mathbf{2 d}$ underwent degradation to a mixture of undetermined products, which had also occurred in the absence of the complex. For the reaction of $\mathbf{1} \cdots \mathbf{2} \mathbf{f} \mathbf{C B}[\mathbf{8}]$, LCMS analysis revealed a species with $\mathrm{m} / \mathrm{z}$ consistent with the desired cross-[2+2] product, $\mathbf{3 f}$ (Scheme 1).

To verify the identity and investigate the stereochemistry of the adduct between $\mathbf{1}$ and $\mathbf{2} \mathbf{f}$, we subjected the crude reaction mixture after 2 days of irradiation to semi-prep HPLC purification to isolate adduct $\mathbf{3 f}$. We confirmed the syn stereochemistry of the product by 2D-NOESY NMR and by the large splitting of the cyclobutane protons, which is predicted by the Karplus relationship (see Figures S28-S31). The syn stereochemistry is consistent with the expected face-to-face $\pi$ - $\pi$ interaction of the stilbenes in the complexes. The primary side product was 4-carboxybenzaldehyde, 4 , which we isolated via HPLC and assigned via mass spectrometry, ${ }^{1} \mathrm{H}$ NMR, and ${ }^{13} \mathrm{C}$ NMR spectroscopies (see Figures S32-34). This compound is likely formed through oxidative cleavage of 7 . Based on previously reported oxidations of stilbenes in water, we believe that this product is formed via diffusion of $2 \mathbf{f}^{+}$ away from $\mathbf{1}^{\mathbf{0}^{+}}$following the initial CT event. ${ }^{4,47,48} \mathbf{2 \mathbf { f } ^ { + }}$ is attacked by water then further oxidized by $\mathbf{1}$ or its derivatives to form a diol, $\mathbf{5}$, which is oxidatively cleaved to the benzaldehyde (Scheme $\mathbf{1})$. In support of this hypothesis, subjecting an authentic sample of diol $\mathbf{5}(1: 1 \mathrm{dr})$ to the reaction conditions yielded exclusively 4 . Rigorous exclusion of oxygen via freeze-pump-thaw cycles and reaction under argon did not suppress formation of the benzaldehyde byproduct, supporting the role of 1 and its derivatives as terminal oxidants in these unwanted reactions (see SI). Irradiation of $\mathbf{2 f}$ with 400 or 350 $\mathrm{nm}$ light in the absence of $\mathbf{1}$ and $\mathbf{C B}[\mathbf{8}]$ led only to cis-trans isomerization.

A control reaction between 1 and $2 \mathbf{f}$ conducted without $\mathbf{C B}$ [8] led to greater production of benzaldehyde byproduct 4 in addition to two new byproducts. Using LCMS and ${ }^{1} \mathrm{H}$ NMR, these new byproducts were determined to be $\mathbf{3} \mathbf{g}$, the cyclobutane produced by [2+2] dimerization of $\mathbf{2} \mathbf{f}$, and $\mathbf{3 h}$, an asymmetric cyclobutane containing three $p$-benzoate groups and one methylpyridinium (see Figures S35 - S38). Side products 4 and $\mathbf{3} \mathbf{g}$ would both be produced via diffusion of $\mathbf{2} \mathbf{f}^{+}$away from the initial CT complex. Benzaldehyde 4 arises through the oxidative pathway described above, while cyclobutane $\mathbf{3} \mathbf{g}$ arises from attack by a second molecule of $\mathbf{2 f}$. The asymmetric cyclobutane product, $\mathbf{3 h}$, could arise via retro-[2+2] of $\mathbf{3} \mathbf{f}$ to produce an asymmetric cis-stilbene, $\mathbf{5}$, which then reacts with $\mathbf{2} \mathbf{f}$ or $\mathbf{2} \mathbf{f}^{+}$. UVVis spectroscopy of $\mathbf{3} \mathbf{f}$ reveals an absorption tail in the visible region, such that retro-[2+2] could plausibly be slowly initiated by the $400 \mathrm{~nm}$ LEDs (Figure S7). Identification of these products reveals that $\mathbf{C B}[8]$ acts not just to pre-assemble $\mathbf{1}$ and $\mathbf{2 f}$ into a CT complex prior to irradiation, but also to prevent diffusion of reactive intermediates produced by irradiation, including $\mathbf{2} \mathbf{f}^{+}$and the asymmetric stilbene produced by retro-[2+2]. A similar protective role for a macrocyclic host has previously been demonstrated for a pillar[6]aren-azastilbene system. ${ }^{1,49}$ 


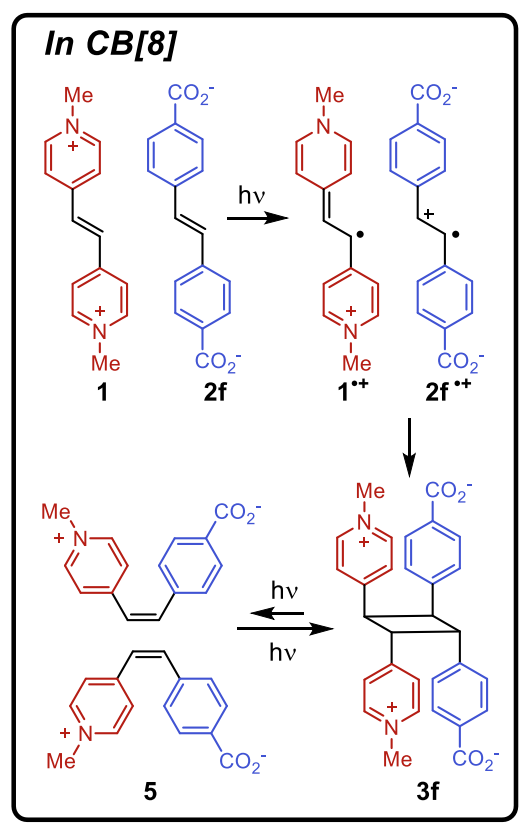

Outside of $\mathrm{CB}[8]$

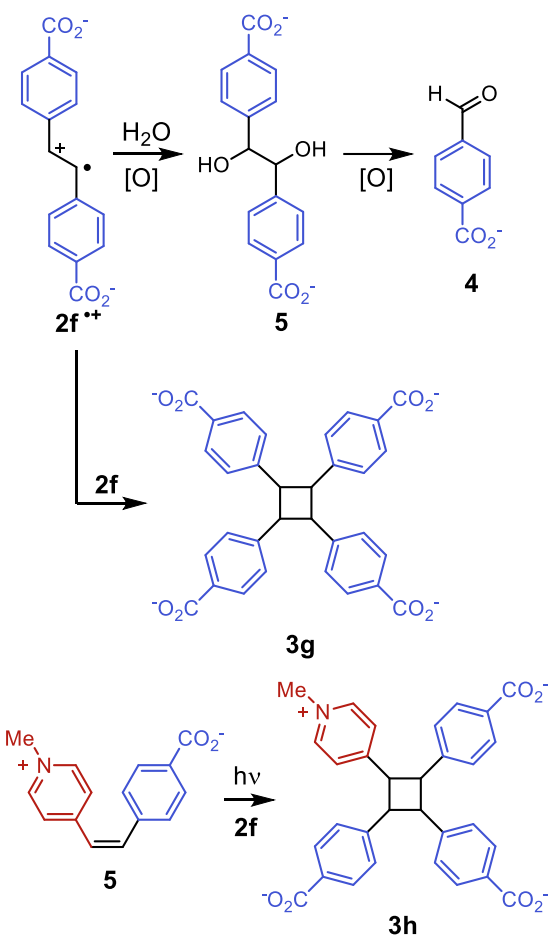

Scheme 1. Proposed mechanism for the reaction of $\mathbf{1}$ and $\mathbf{2 f}$. The upper portion are steps facilitated by the donor-acceptor host-guest interaction inside of $\mathbf{C B}$ [8]. Steps outside of $\mathbf{C B}$ [8] are suppressed by removal of free $\mathbf{2} \mathbf{f}$ by $\mathbf{C B}[\mathbf{8}]$. The relative stereochemistries of $\mathbf{3} \mathbf{g}$ and $\mathbf{3 h}$ were not determined.

To validate that the [2+2] reaction between $\mathbf{1}$ and $\mathbf{2} \mathbf{f}$ occurs via $\mathrm{CT}$, we tested the reaction under various conditions (Table 2). We found that irradiation of a $15 \mathrm{mM}$ solution of the CT complex with $400 \mathrm{~nm}$ LEDs in $0.1 \mathrm{M} \mathrm{Cs}_{2} \mathrm{CO}_{3}$ provides the highest yield of $\mathbf{3 f}(67 \%$, Table 2, Entry 1, see Experimental Section for details). Irradiation with higher-energy 350-nm light led to a decreased yield 
of $\mathbf{3 f}$ relative to $\mathbf{4}$, likely due to increased excitation of free $\mathbf{2} \mathbf{f}$ instead of the CT complex (Entry 2 ). The reaction could proceed under $470 \mathrm{~nm}$ irradiation, albeit with slightly lower yield compared to 400 $\mathrm{nm}$, while lower-energy light provided very little product (Entries 3 and 4). Longer irradiation times did not change the product distribution or yields. Light was required for both desired and side reactions to occur (Entry 5). These irradiation experiments suggest that the [2+2] reaction between 1 and $\mathbf{2} \mathbf{f}$ is initiated by CT. Consistent with the contributions of supramolecular and diffusion processes in this reaction, concentration had a significant effect on reaction efficiency, with $15 \mathrm{mM}$ conditions leading to a higher yield of $\mathbf{3} \mathbf{f}$ relative to $1.5 \mathrm{mM}$ conditions (Entry 6). As discussed above, $\mathbf{C B}$ [8] improves the selectivity for and yield of $\mathbf{3} \mathbf{f}$ (Entry 8$)$.
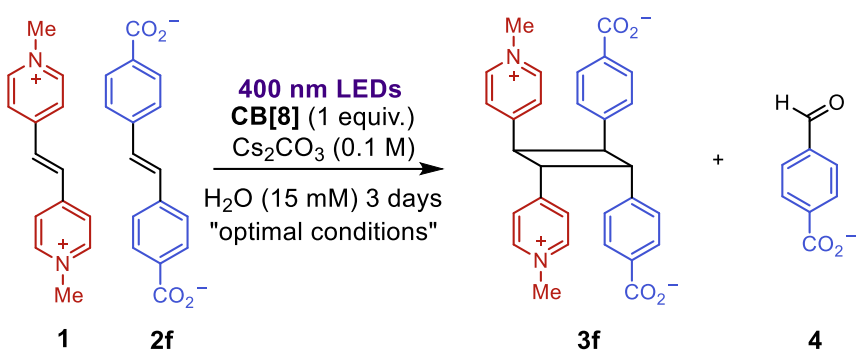

\begin{tabular}{|c|c|c|c|}
\hline Entry & Deviation from optimal conditions & Yield of $\mathbf{3 f}(\%)$ & Yield of $\mathbf{4}(\%)$ \\
\hline $\mathbf{1}$ & none & $\mathbf{6 7}$ & $\mathbf{2 0}$ \\
\hline 2 & $350 \mathrm{~nm}$ lamps & 31 & 30 \\
\hline 3 & Blue (470 nm) LEDs & 55 & 28 \\
\hline 4 & Green (525 nm) LEDs & 9 & 5 \\
\hline 5 & No light & 0 & 0 \\
\hline 6 & $1.5 \mathrm{mM}$ & 23 & 37 \\
\hline 7 & $\mathrm{Na}_{2} \mathrm{CO}_{3}$ instead of $\mathrm{Cs}_{2} \mathrm{CO}_{3}$ & 13 & 5 \\
\hline 8 & No CB $[8]$ & 20 & 16 \\
\hline
\end{tabular}

Table 2. Yield of desired cyclobutane product $3 \mathrm{f}$ and undesired aldehyde side product 4 under various reaction conditions. The optimal conditions are indicated in the chemical equation above the table. Yields determined by LCMS with an internal standard of butylhydroxytoluene (see SI for details). All values represent the average of two experiments, with a range of less than $5 \%$ for all cases except for entry 5 , which has a range of $15 \%$.

\section{DISCUSSION}

Having confirmed that $\mathbf{1}$ and $\mathbf{2} \mathbf{f}$ engage in a CT-initiated cross-[2+2] reaction, we sought to understand why this was the only pair of substrates that exhibited the desired photoreactivity despite the similar complexation behavior exhibited by other stilbenes. We modelled the ternary complexes of $\mathbf{2 a}, \mathbf{2 b}, \mathbf{2 c}$, and $\mathbf{2 f}$ with $\mathbf{1}$ and $\mathbf{C B}$ [8] using DFT using B3LYP functional, 6-31G(d) basis set, and a CPCM $\mathrm{H}_{2} \mathrm{O}$ solvent model, which have been used to model donor-acceptor host-guest ternary complexes with $\mathbf{C B}$ [8] before (see Computational Methods). ${ }^{34}$ The energy-minimized structures all exhibited similar geometries, with the alkenes nearly parallel $\left(<15^{\circ}\right)$ and well within the $4.2 \AA$ distance defined by the Schmidt criterion for $[2+2]$ reactions, ${ }^{12}$ indicating that the differences in reactivity were not due to the geometry of the CT complexes (Figure 3a). 
We hypothesized that the primary pathway that competes with the desired [2+2] reaction following CT excitation is back electron transfer (BET). To investigate this hypothesis, we measured the rates of BET following CT using transient absorption (TA) spectroscopy (Figure 3b). We found

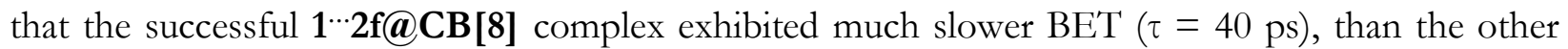
pairs ( $\tau$ between $<0.3$ and $10 \mathrm{ps).} \mathrm{The} \mathrm{relatively} \mathrm{longer} \mathrm{lifetime} \mathrm{of} \mathbf{1}^{\mathbf{*}}$ and $\mathbf{2 \mathbf { f } ^ { + }}$ indicates that the [2+2] reaction is quite slow, and is consistent with the role of $\mathbf{C B}[8]$ in protecting these intermediates.

What is the origin of the dramatic differences in BET, and thus reactivity? We turned to the classical Marcus relationship, which relates the rate of electron transfer, $k \mathrm{BET}$, to the associated free energy change according to Eq. 1:

$$
k_{\mathrm{BET}}=\frac{k_{B} T}{h} e^{-\frac{\Delta G^{\ddagger}}{R T}}
$$

where $k_{B}$ is Bolzmann's constant, $T$ is temperature, $h$ is Planck's constant, $R$ is the gas constant and $\Delta G^{\ddagger}$ is the free energy of activation given by Eq. 2 .

$$
\Delta G^{\ddagger}=\frac{\lambda}{4}\left(1+\frac{\Delta G_{\mathrm{BET}}}{\lambda}\right)^{2}
$$

In Eq. $2, \lambda$ is the reorganization energy and $\Delta G_{\mathrm{BET}}$ is the free energy change of the back electron transfer. $\Delta G_{\mathrm{BET}}$ represents a combination of the energy difference between the LUMO of one molecule and the HOMO of the other, $\Delta E_{\mathrm{CT}}$, and the Coulombic work associated with charge separation and recombination (see SI for a full derivation). The parameters that change between our various substrate pairs are $\Delta E_{\mathrm{CT}}$ and the charge of the electron-rich stilbene. The effect of different $\Delta E_{\mathrm{CT}}$ produces the well-known Marcus curve, wherein electron transfer is accelerated by greater CT energy changes up until the reorganization energy, after which greater free energy changes lead to deceleration in the "Marcus inverted region". The effect of charge can be understood qualitatively. If the CT state has weaker electrostatic stabilization than the ground state, then BET will be more favorable than would be predicted based solely on CT energy as calculated using the energy levels of isolated substrates or measured by UV-Vis. 
a

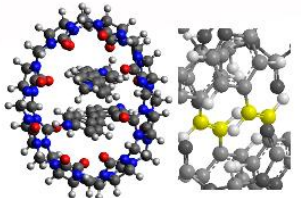

1.2a@CB[8]

$14^{\circ}, 3.5 \AA$

b

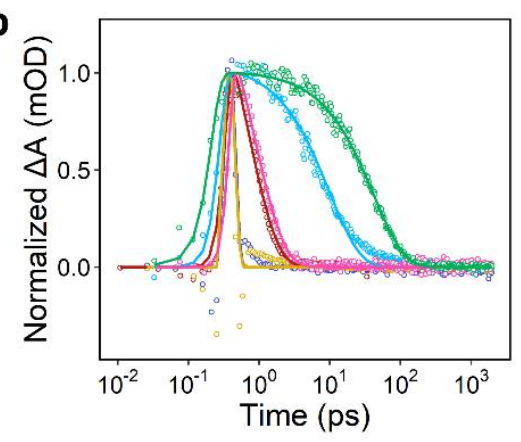

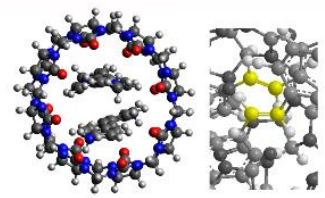

1-.2b@CB[8]

$11^{\circ}, 4.0 \AA$

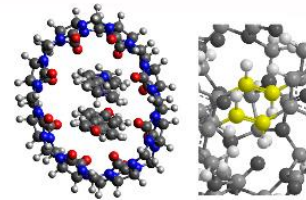

1-.2c@CB[8]

$1^{\circ}, 3.5 \AA$

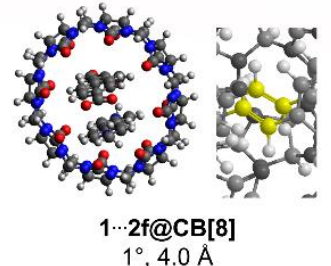

c

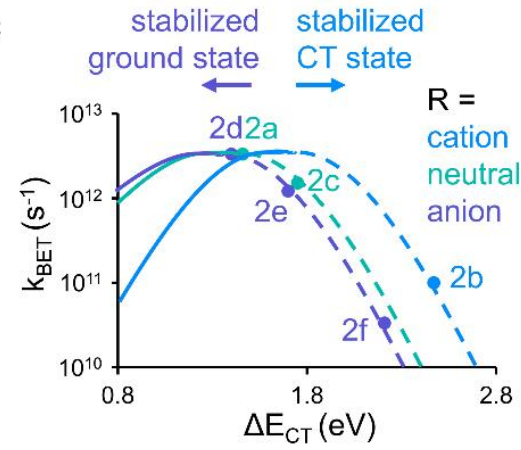

d

-1“2a@CB[8]

- 1“2b@CB[8]

- 1'2c@CB[8]

- 1.2d@CB[8]

$-12 \mathrm{e}$

- 1 2f@CB[8]

$\mathrm{R}=$ neutral $(2 \mathrm{a}, 2 \mathrm{c})$

Electrostatics weakly stabilize ground state
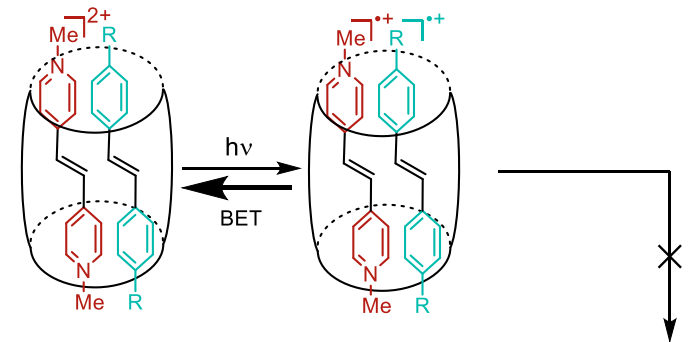

$\mathrm{R}=\operatorname{anion}(2 \mathrm{~d}, 2 \mathrm{e}, 2 \mathrm{f})$ Electrostatics strongly stabilize ground state

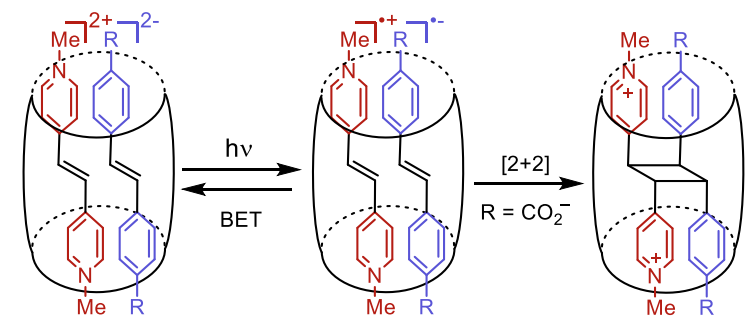

$\mathrm{R}=$ cation $(2 \mathrm{~b})$

Electrostatics destabilize ground state
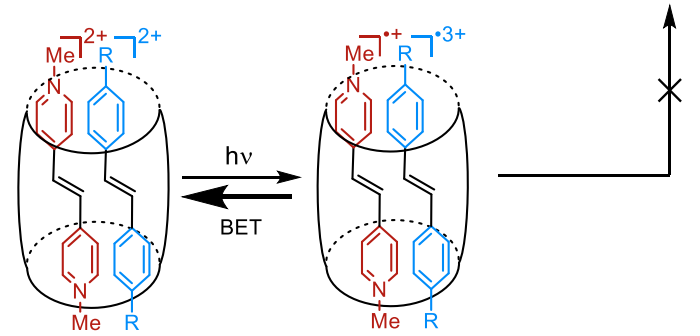
Figure 3. (a) DFT-computed energy-minimized structures of several complexes. The images on the right highlight the potentially reactive double bonds in yellow, and the angle and distances below each structure indicate the angle and distance between them. (b) BET monitored by transient absorption spectroscopy following excitation of the charge transfer (CT) complexes in water $(7.5 \mathrm{mM})$. The CT band of the complexes were excited with a pump pulse $(540 \mathrm{~nm}$ for $\mathbf{2 a}$ and $\mathbf{2 d}, 490 \mathrm{~nm}$ for $\mathbf{2 c}$, and $440 \mathrm{~nm}$ for all others) and timeresolved optical spectra were measured with a delayed pump pulse. The plot shows the decay of the signal of the singly oxidized stilbenes $(600 \mathrm{~nm}$ for $2 \mathrm{a}, 550 \mathrm{~nm}$ for $2 \mathbf{c}, 540 \mathrm{~nm}$ for $2 \mathrm{~d}, 490 \mathrm{~nm}$ for $2 \mathrm{e}$, and $480 \mathrm{~nm}$ for $\mathbf{2 f}$ ). The solid curves show mono-exponential fits. (c) Electron transfer rate vs. charge transfer energy determined by UV-vis absorption onset. Labels next to each data point indicate the electron donor. The lines qualitatively represent the Marcus relationship predicted by Eq. 1-4. The position of the Marcus curve along the $\mathrm{x}$-axis depends on the charge of the donor stilbene, while the position of each data point on the curve is determined by the CT energy of the corresponding donor-acceptor pair. Dotted lines indicate the Marcus inverted region. (d) CT excitation produces radical pairs that can either react in a formal [2+2] cycloaddition or relax through back electron transfer (BET) to the ground state. The relative electrostatic stabilities of the $\mathrm{CT}$ and ground states are determined by the charge (anionic, neutral, or cationic) of the electron donor.

The fact that the substrate pair with the slowest BET also has the greatest CT energy as measured by UV-vis suggests that the BET process falls within the Marcus inverted region, where greater energy differences between the charge separated and ground state serve to slow down BET. A plot of BET rate determined from TA vs. CT energy determined from the onsets of UV-vis absorption indeed reveals decreased BET rate with greater CT energy (Figure 3c). This Marcus inversion effect is enhanced by the differences in electrostatic interactions between the various substrate pairs (Figure 3d). Weaker electrostatic interactions in the CT state relative to the ground state push the BET process of the $1 \cdots 2 \mathbf{f} @ \mathbf{C B}[8]$ deeper into the Marcus inverted region. This effect also explains why the $1 \cdots 2 \mathbf{b} @ \mathbf{C B}[\mathbf{8}]$, which has a very similar CT energy to the successful pair, has slower BET: electrostatic interactions in this pair make the free energy change of BET less negative, accelerating it by pulling it out of the Marcus inverted region. These trends are depicted qualitatively by the lines in Figure 3c.

We believe that the role of electrostatics in slowing down BET will be critical to the development and application of CT-initiated cross-photocycloadditions. Among the para-substituted symmetric stilbenes we studied, $\mathbf{1}$ and $\mathbf{2} \mathbf{f}$ were a uniquely productive pair for the cross-[2+2] photoreaction. On the one hand, small CT gaps are favored since irradiating CT bands selectively requires them to be in the visible, with red-shifted CT excitations being desirable. However, smaller CT gaps pull BET out of the Marcus inverted region, leading to faster BET that outcompetes the desired reaction. A possible solution to this conundrum is to introduce charge to the substrates, which pushes BET back into the Marcus inverted region via the electrostatic terms in Eq. 3. However, introducing too much negative charge to a substrate shuts down host-guest interactions due to repulsion by the partially negatively charged $\mathbf{C B}[8]$ portal, as is the case with $\mathbf{2 e}$. Thus, $\mathbf{2} \mathbf{f}$ appears to possess a delicate balance of energy levels and charge such that it can engage in ternary complexation with 1 and $\mathbf{C B}$ [8], form a CT complex in the visible range, and undergo cycloaddition competitive with BET. 
Intrigued by the potentially unique reactivity of $\mathbf{1}$ and $\mathbf{2} \mathbf{f}$, we used DFT to calculate the electronic structure of 36 different para-substituted symmetric stilbenes, then used Eqs. 1-4 to predict the relative BET rates between 1 and each stilbene following charge transfer (see SI for DFT results). We estimated the reorganization energy and dielectric constant within $\mathbf{C B}$ [8] based on previous studies of electron transfer in $\mathbf{C B}[8] .{ }^{44,50}$ We emphasize that these calculations were not meant to quantitatively predict BET rates, but rather to compare BET rates in possible stilbene pairs. No neutral stilbenes exhibited both visible CT (i.e. $<2.8 \mathrm{eV}$ CT gap) and relatively slow BET. The only anionic stilbenes that met these criteria were $\mathbf{2} \mathbf{f}$, other more highly charged anionic substrates such as $\mathbf{2 e}$, and a sulfonate-substituted stilbene $\mathbf{2} \mathbf{g}$. We synthesized $\mathbf{2} \mathbf{g}$ (see Experimental Section), but it produced an insoluble complex with 1 and $\mathbf{C B}$ [8], preventing further study. Our predictions, along with the experimental results of $\mathbf{2} \mathbf{e}$ as representative highly charged anion, suggest that $\mathbf{2} \mathbf{f}$ is the only parasubstituted symmetric stilbene among the 36 we modeled able to undergo host-guest templated, CTinitiated cross-[2+2] with 1 .

\section{CONCLUSION}

We hypothesized that donor-acceptor host-guest ternary complexes could template [2+2] photocycloadditions to achieve selective cross-reactivity. We investigated a series of model stilbene substrates and found that although many pairs formed the desired ternary complexes, only one exhibited the desired photoreactivity following CT excitation. We attribute this divergent reactivity to highly variable BET rates measured by transient absorption spectroscopy. The optimal pair of donor and acceptor stilbenes exhibit relatively slow back electron transfer following CT, allowing the desired reaction to occur before charge recombination. This slow BET, in turn, is a product of the energy levels and complementary charges of the substrates, which stabilize the ground state relative to the CT state and thus slow down BET via Marcus inversion. The host-guest interaction provided by $\mathbf{C B}$ [8] not only templates the reaction, but also serves to protect the initially generated radical ions following CT from side reactions. This work illustrates the beneficial role that host-guest chemistry can play in discovering and optimizing CT-initiated photochemistry. 


\section{EXPERIMENTAL SECTION}

General Section. Unless otherwise noted, reactions were performed under $\mathrm{N}_{2}$ atmosphere in ovendried $\left(150^{\circ} \mathrm{C}\right)$ glassware. Reaction progress was monitored by thin layer chromatography (EMD 250 $\mu \mathrm{m}$ silica gel 60-F254 plates) or by liquid chromatography-mass spectrometry using a Agilent 6120 Quadrupole LC/MS. Automated column chromatography was performed using SiliCycle SiliaFlash F60 (40-63 $\mu \mathrm{m}, 60 \AA)$ in SNAP cartridges on a Biotage Isolera One. Organic solvents were removed in vacuo using a rotary evaporator (Büchi Rotovapor R-100, 20-200 torr) and residual solvent was removed under high vacuum $(<0.1$ torr). Commercial reagents and solvents were purchased from Sigma-Aldrich, Acros, Alfa Aesar, TCI, or Oakwood and used as received. DMF and dichlormethane were purified and dried using a solvent-purification system that contained activated alumina then degassed with $\mathrm{N}_{2}$ sparging prior to use. Proton nuclear magnetic resonance ( ${ }^{1} \mathrm{H}$ NMR) spectra, carbon nuclear magnetic resonance $\left({ }^{13} \mathrm{C} \mathrm{NMR}\right)$, and phosphorous nuclear magnetic resonance $\left({ }^{31} \mathrm{P} \mathrm{NMR}\right)$ spectra were recorded on Bruker AVANCE-500 spectrometers at $500 \mathrm{MHz}$ and $125 \mathrm{MHz}$, and referenced to the solvent residual peaks. NMR data are represented as follows: chemical shift ( $\delta \mathrm{ppm})$, multiplicity ( $\mathrm{s}=$ singlet, $\mathrm{d}=$ doublet, $\mathrm{t}=$ triplet, $\mathrm{q}=$ quartet, $\mathrm{m}=$ multiplet), coupling constant in Hertz (Hz), integration. UV-vis spectra were collected on a Cary 5000 or Cary 3500 UV-vis-NIR spectrophotometer with an Hg lamp; cuvettes were 10-mm or 2-mm path length quartz cells (Starna 23-Q-10 or 23-Q-2). High-resolution mass spectra were recorded on an Agilent 6210A LC-TOF mass spectrometer. Red $(626 \mathrm{~nm}, 6.6 \mathrm{~W})$, amber $(605 \mathrm{~nm}, 2.4 \mathrm{~W})$, green $(525 \mathrm{~nm}, 5.7 \mathrm{~W})$, and violet (400 $\mathrm{nm}$, power $=6.1 \mathrm{~W})$ LEDs were purchased from superbrightleds.com. See Figures SX-SX for emission profiles of the LEDs. $350 \mathrm{~nm}$ irradiation was provided by ten Rayonet RPR-3500A lamps (power $=8 \mathrm{~W}$ ). Semi-prep HPLC was performed using a Shimadzu LC-6A with a C18 column and a water/acetronitrile mobile phase with $0.1 \%$ trifluoroacetic acid. LCMS was performed on an Agilent 6120 Quadrupole LC/MS using a 2.1 X 50 mm "Poroshell 120" C18 column with a water/acetonitrile mobile phase with $0.1 \%$ formic acid.

\section{Synthesis of starting materials}

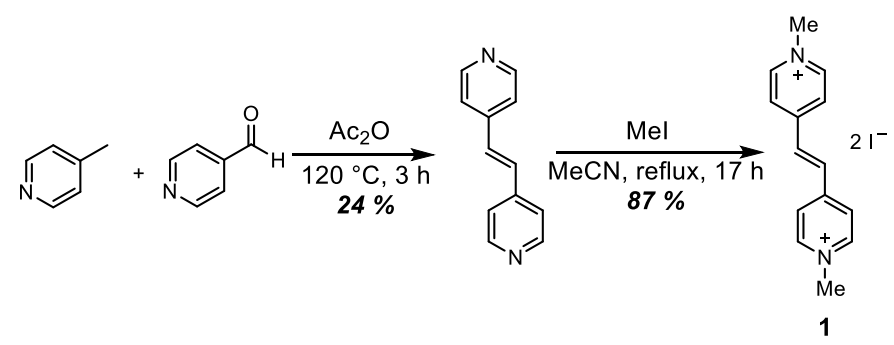

Scheme 2. Synthesis of 1.

(E)-1,2-di(pyridin-4-yl)ethene: Based on a literature procedure, ${ }^{51}$ 4-methylpyridine (4.89 mL, 50.3 mmol), 4-pyridinecarboxaldehyde $(4.73 \mathrm{~mL}, 50.3 \mathrm{mmol})$, and acetic anhydride $(4.81 \mathrm{~mL}, 50.9 \mathrm{mmol})$ were combined in a non-dried $100 \mathrm{~mL}$ round bottom flask with a magnetic stir bar. The reaction was 
heated to $120^{\circ} \mathrm{C}$ and stirred for 3 hours under nitrogen. The reaction was then cooled to room temperature an aqueous solution of $\mathrm{NaOH}(4.0 \mathrm{~g}$ in $40 \mathrm{~mL}$ water) was added to the brown mixture. A dark red solid was collected via vacuum filtration. The solid was recrystallized from an ethanol/water mixture ( $9 \mathrm{~mL}$ ethanol, $35 \mathrm{~mL}$ water) and dried in a vacuum desiccator to yield the product as an orange solid ( $2.18 \mathrm{~g}, 24 \%$ yield). ${ }^{1} \mathrm{H}$ NMR matched the literature report. ${ }^{51}{ }^{1} \mathrm{H}$ NMR $\left(400 \mathrm{MHz}, \mathrm{CDCl}_{3}\right) \delta 8.63(\mathrm{~d}, J=6.2 \mathrm{~Hz}, 3 \mathrm{H}), 7.39(\mathrm{~d}, J=6.2 \mathrm{~Hz}, 3 \mathrm{H}), 7.21$ (s, 2H).

(E)-4,4'-(ethene-1,2-diyl)bis(1-methylpyridin-1-ium) iodide (1): Following a literature procedure, ${ }^{52}(E)-1,2$-di(pyridin-4-yl)ethene $(218 \mathrm{mg}, 1.20 \mathrm{mmol}$ ) was dissolved in $3 \mathrm{~mL}$ of acetonitrile in a non-dried $10 \mathrm{~mL}$ round bottom flask fitted with a reflux condenser and a magnetic stir bar. Methyl iodide $(2.21 \mathrm{~g}, 0.968 \mathrm{~mL}, 15.6 \mathrm{mmol})$ was added and the reaction was heated to reflux under nitrogen for 17 hours. The resulting orange mixture was then cooled and an orange solid was recovered by vacuum filtration. Washing with acetonitrile yielded the product as a chalky orange solid (485 $\mathrm{mg}, 87$ \% yield). ${ }^{1} \mathrm{H}$ NMR in $\mathrm{D}_{2} \mathrm{O}$ matched the literature report. ${ }^{52}{ }^{1} \mathrm{H}$ NMR $\left(500 \mathrm{MHz}, \mathrm{D}_{2} \mathrm{O}\right) \delta 8.79(\mathrm{~d}, J=$ $6.8 \mathrm{~Hz}, 4 \mathrm{H}), 8.24$ (d, J = 6.9 Hz, 4H), 7.89 (s, 2H), 4.39 (s, 6H).

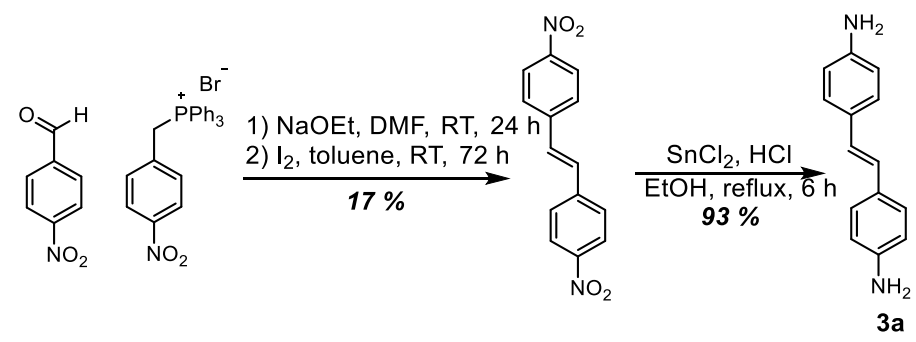

Scheme S2. Synthesis of 2a.

(E)-1,2-bis(4-nitrophenyl)ethene: Following a literature procedure, ${ }^{53}$ (4nitrobenzyl)triphenylphosphonium bromide (5.00 g, $10.5 \mathrm{mmol}$ ), 4-nitrobenaldehyde (1.58 g, 10.5 $\mathrm{mmol}$ ) and sodium ethoxide $(925 \mathrm{mg}, 13.6 \mathrm{mmol})$ were added to an oven-dried $250 \mathrm{~mL}$ round bottom flask with a magnetic stir bar. Dry DMF was added and the red solution was stirred at room temperature under nitrogen. After 24 hours, the reaction mixture was filtered and the filtrate was collected. Solvent was removed in vacuo to provide a bright yellow solid, which was recrystallized twice from ethanol. Comparison to the literature ${ }^{1} \mathrm{H}$ NMR revealed that we had produced the undesired $Z$ isomer. ${ }^{53}{ }^{1} \mathrm{H}$ NMR $\left(500 \mathrm{MHz}, \mathrm{CDCl}_{3}\right) \delta 8.12(\mathrm{~d}, J=8.9 \mathrm{~Hz}, 4 \mathrm{H}), 7.35(\mathrm{~d}, J=8.8 \mathrm{~Hz}, 4 \mathrm{H}), 6.84$ (s, $2 \mathrm{H})$. We produced the desired E-isomer via iodine-catalyzed isomerization. The Z-isomer was suspended in $20 \mathrm{~mL}$ of toluene and two crystals of iodine were added. The mixture was stirred at room temperature under nitrogen. Isomerization was monitored using ${ }^{1} \mathrm{H}$ NMR. After 72 hours, the isomerization was complete. The reaction mixture was diluted in $100 \mathrm{~mL}$ of ethyl acetate and washed with $100 \mathrm{~mL}$ of aqueous sodium thiosulfate $(10 \mathrm{wt} \%)$. The aqueous layer was removed and the yellow solid suspended in the organic layer was collected via vacuum filtration. This solid was washed with hexanes and dried on high vacuum to provide the desired product ( $485 \mathrm{mg}, 17 \%$ yield). ${ }^{1} \mathrm{H} \mathrm{NMR}$ 
matched the literature report. ${ }^{53}{ }^{1} \mathrm{H}$ NMR $\left(500 \mathrm{MHz}, \mathrm{CDCl}_{3}\right) \delta 8.27(\mathrm{~d}, J=8.9 \mathrm{~Hz}, 4 \mathrm{H}), 7.69(\mathrm{~d}, J=$ $8.9 \mathrm{~Hz}, 1 \mathrm{H}), 7.30$ (s, 1H).

(E)-4,4'-(ethene-1,2-diyl)dianiline (2a): Following a literature procedure, ${ }^{53}$ (E)-1,2-bis(4nitrophenyl)ethene $(200 \mathrm{mg}, 0.740 \mathrm{mmol})$ and $\mathrm{SnCl}_{2}(1.67 \mathrm{~g}, 7.40 \mathrm{mmol})$ were suspended in a mixture of ethanol $(10 \mathrm{~mL})$ and hydrochloric acid $(3 \mathrm{~mL})$ in a round bottom flask equipped with a magnetic stir bar. The reaction was stirred under $\mathrm{N}_{2}$ for one hour then heated to reflux for six hours. The reaction mixture was then poured onto ice and the $\mathrm{pH}$ was adjusted to $\sim 8$ using a sodium hydroxide solution. The resulting mixture was extracted with ethyl acetate $(3 \times 20 \mathrm{~mL})$, and the combined organic layers were dried of $\mathrm{MgSO}_{4}$. Filtration and removal of the solvent in vacuo provided the product as a light orange solid (145 mg, $93 \%$ yield). ${ }^{1} \mathrm{H}$ NMR matched the literature report. ${ }^{53}{ }^{1} \mathrm{H} \mathrm{NMR}(500 \mathrm{MHz}$, DMSO-d $d_{6} \delta 7.26-7.02(\mathrm{~m}, 4 \mathrm{H}), 6.71(\mathrm{~s}, 2 \mathrm{H}), 6.57-6.47(\mathrm{~m}, 4 \mathrm{H}), 5.13(\mathrm{~s}, 4 \mathrm{H})$.

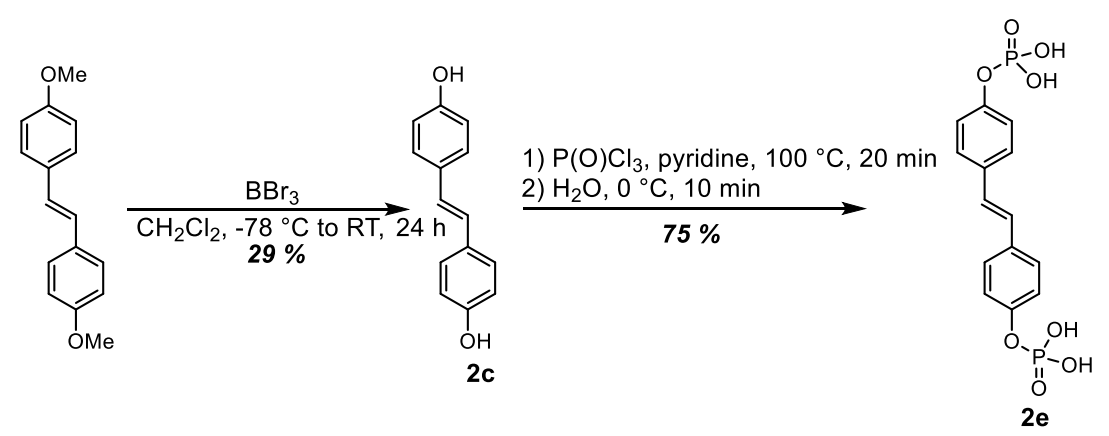

Scheme S3. Synthesis of the $2 \mathrm{c}$ and $2 \mathrm{e}$.

(E)-4,4'-(ethene-1,2-diyl)diphenol (2c): (E)-1,2-bis(4-methoxyphenyl)ethene (2.40 g, $9.99 \mathrm{mmol})$ was dissolved in $100 \mathrm{~mL}$ of dry dichloromethane in a flame-dried $200 \mathrm{~mL}$ Schlenk flask. The resultant yellow slurry was cooled to $-78^{\circ} \mathrm{C}$ in a dry ice/acetone bath. Tribromoborane $(2.84 \mathrm{~mL}, 30.0 \mathrm{mmol})$ was added over five minutes, causing an immediate darkening and precipitation of a white solid. The reaction was stirred under nitrogen while the bath slowly warmed overnight. After 24 hours, the reaction was quenched by slow addition of $100 \mathrm{~mL}$ of ice-cold water. $200 \mathrm{~mL}$ of ethyl acetate were added and the oraganic layer was separated and washed with brine. The solvent was removed in vacuo and the residue was purified via column chromatography (hexanes/ethyl acetate; 2 to $45 \%$ linear gradient) to provide the produce as a white solid (624 mg, $29 \%$ yield). ${ }^{1} \mathrm{H}$ NMR matched the literature report. ${ }^{54}{ }^{1} \mathrm{H}$ NMR $\left(500 \mathrm{MHz}\right.$, Acetone- $\left.d_{6}\right) \delta 7.44-7.36(\mathrm{~m}, 4 \mathrm{H}), 6.96(\mathrm{~s}, 2 \mathrm{H}), 6.86-6.79(\mathrm{~m}, 4 \mathrm{H})$.

(E)-4-(4-(phosphonooxy)styryl)phenyl dihydrogen phosphate (2e): (E)-4,4'-(ethene-1,2diyl)diphenol (255 mg, $1.20 \mathrm{mmol}$ ) was dissolved in $1 \mathrm{~mL}$ of pyridine to produce a yellow solution. Addition of this solution to phosphoryl trichloride $(1.12 \mathrm{~mL}, 12.0 \mathrm{mmol})$ led to an exothermic reaction and a white precipitate. The reaction was heated to $100{ }^{\circ} \mathrm{C}$ and stirred under nitrogen for 20 minutes. The reaction mixture was then cooled to $0{ }^{\circ} \mathrm{C}$ in an ice bath and water $(300 \mathrm{uL})$ was slowly added to hydrolyze the phosphoryl chlorides. The resultant white slurry was basified with $1 \mathrm{M} \mathrm{NaOH}$ to a pH of $\sim 10$. The aqueous solution was washed with $5 \times 10 \mathrm{~mL}$ of ethyl acetate, then acidified with concentrated $\mathrm{HCl}$ to produce a white precipitate, which was collected via vacuum filtration, washed with water, and dried under high vacuum to provide the product (333 mg, $75 \%$ yield). ${ }^{1} \mathrm{H}$ NMR (400 
$\left.\mathrm{MHz}, \mathrm{DMSO}-d_{6}\right) \delta 7.34(\mathrm{~d}, J=8.3 \mathrm{~Hz}, 4 \mathrm{H}), 7.05-6.98(\mathrm{~m}, 6 \mathrm{H}) \cdot{ }^{13} \mathrm{C}\left\{{ }^{1} \mathrm{H}\right\}$ NMR $(126 \mathrm{MHz}, \mathrm{DMSO})$ $\delta 151.49(\mathrm{~d}, J=6.4 \mathrm{~Hz}), 132.90,127.57,127.08,120.46$ (d, $J=5.0 \mathrm{~Hz}) \cdot{ }^{31}$ P NMR $(202 \mathrm{MHz}, \mathrm{DMSO})$ $\delta$-5.16 HRMS (ESI-ToF): $m / z=-392.9911$ (calc'd for $[\mathrm{M}-2 \mathrm{H}+\mathrm{Na}]:-392.9911$ ).

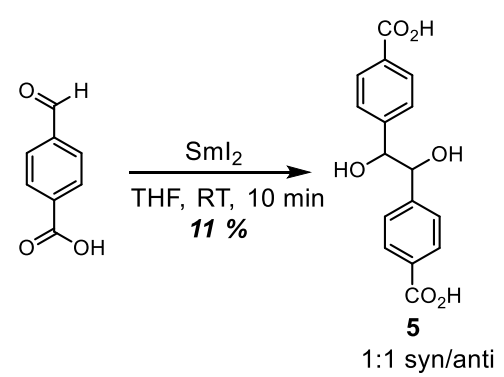

Scheme S4. Synthesis of 5 .

4,4'-(1,2-dihydroxyethane-1,2-diyl)dibenzoic acid (5): Following a literature procedure, ${ }^{55}$ 4Carboxybenzaldehyde (212 mg, $1.41 \mathrm{mmol}$ ) was dissolved in $5 \mathrm{~mL}$ of dry THF then dropwise added to $14.1 \mathrm{~mL}$ of a $0.1 \mathrm{M}$ THF solution of samarium diiodide $(1.41 \mathrm{mmol})$ in a round bottom flask with a stir bar. The solution immediately turned yellow and was stirred under $\mathrm{N}_{2}$ for an additional 10 minutes. Then, about $30 \mathrm{~mL}$ of $0.1 \mathrm{M} \mathrm{HCl}$ were added followed by $30 \mathrm{~mL}$ of ethyl acetate. The organic layer was separated and the aqueous layer was extracted with a further $20 \mathrm{~mL}$ of ethyl acetate. The combined organic layers were washed with aqueous sodium thiosulfate and brine before being dried over $\mathrm{Na}_{2} \mathrm{SO}_{4}$. Filtration and removal of the solvent in vacuo provided a crude product that contained some of the starting material. The desired diol was purified via column chromatography $(20 \%$ methanol in dichloromethane), yielding a white solid that contained a 1:1 mixture of the syn and anti diastereomers (23 mg, $11 \%$ yield). ${ }^{1} \mathrm{H}$ NMR matched a literature report. ${ }^{56}{ }^{1} \mathrm{H} \mathrm{NMR}(500 \mathrm{MHz}$, DMSO-d $\left.d_{6}\right) \delta 7.86-7.80(\mathrm{~m}, 2 \mathrm{H}), 7.85-7.79(\mathrm{~m}, 2 \mathrm{H}), 7.78-7.72(\mathrm{~m}, 2 \mathrm{H}), 7.34(\mathrm{~d}, J=8.3 \mathrm{~Hz}, 2 \mathrm{H})$, $7.22(\mathrm{~d}, J=8.2 \mathrm{~Hz}, 2 \mathrm{H}), 5.58(\mathrm{~s}, 1 \mathrm{H}), 5.48(\mathrm{~s}, 1 \mathrm{H}), 4.73(\mathrm{~d}, J=2.2 \mathrm{~Hz}, 1 \mathrm{H}), 4.66(\mathrm{~d}, J=2.4 \mathrm{~Hz}, 1 \mathrm{H})$.

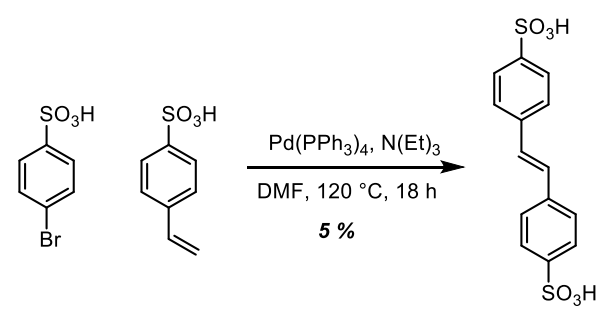

Scheme S4. Synthesis of $2 \mathrm{~g}$.

$2 \mathrm{~g}$

(E)-4,4'-(ethene-1,2-diyl)dibenzenesulfonic acid (2g): Following a modified literature proecedure, ${ }^{57}$ p-bromosulfonic acid hydrate $(510 \mathrm{mg}, 2.00 \mathrm{mmol})$, sodium $p$-vinylbenzenesulfonate (412 mg, $2.00 \mathrm{mmol}$ ), tetrakis(triphenylphosphine)palladium(0) (231 $\mathrm{mg}, 0.200 \mathrm{mmol}$ ), and 
triethylamine $(697 \mu \mathrm{L}, 5.00 \mathrm{mmol})$ were dissolved in dry, $\mathrm{N}_{2}$-sparged dimethylformamide. The mixture was sparged with $\mathrm{N}_{2}$ for 5 minutes before heating to $120{ }^{\circ} \mathrm{C}$ under $\mathrm{N}_{2}$ overnight. After 18 hours, the mixture was cooled and filtered. The residue was collected and dissolved in $5 \mathrm{~mL}$ of hot 1:1 water/ethanol, then this mixture was hot filtered and the filtrate was collected. Solvent was removed in vacuo to yield a grey powder. This powder was suspended in $5 \mathrm{~mL}$ of DMF then re-subjected to the purification above to yield $33 \mathrm{mg}(5 \%)$ of the final lightly grey product. ${ }^{1} \mathrm{H}$ NMR matches the literature report. ${ }^{57}{ }^{1} \mathrm{H}$ NMR (500 MHz, DMSO) $\delta 7.60-7.54(\mathrm{~m}, 8 \mathrm{H}), 7.27$ (s, 2H).

Host-guest binding titrations. To determine the strength of the various host-guest interactions relevant to this work, we used ${ }^{1} \mathrm{H}$ NMR titrations (Figure SX). We prepared solutions of 1 and $1 @ \mathbf{C B}$ [8] in $\mathrm{D}_{2} \mathrm{O}$ at concentrations of 1.0 or $0.5 \mathrm{mM}$ (see Figure SX caption). For each experiment, we split a sample into two parts and added an excess ( 5 times) of the second substrate (the "guest") to one of the portions. We then iteratively added small amounts of this second portion to the first and measured ${ }^{1} \mathrm{H}$ NMR after each addition. For some samples, this led to a gradual shifting of 1 peaks, which we could then track (indicated by "Chemical Shift" y-axes). For others, exchange between bound and unbound states was slow on the NMR time scale so new sets of peaks emerged upon addition of the "guest", which we could integrate to determine the concentration of bounds species (indicated by "Conc. complex" y-axes). In all cases, we fit the experimental data to a 1:1 binding model using supramolecular.org. ${ }^{46}$ For some of the interactions, the data appear linear, which precludes accurate fitting. Based on the concentrations at which we performed these experiments, we estimate that these samples exhibit binding constants of a greater order of magnitude than $10^{5} \mathrm{M}^{-1}$. For $2 \mathbf{a}$ and 2c, analysis is further complicated by the insolubility of these molecules in $\mathrm{D}_{2} \mathrm{O}$, such that we can only observe the bound "guest" in our experiments. This observation indicates that the binding constant of these stilbenes in very great, indeed. Using the same titration experiment, $\mathbf{3} \mathbf{f}$ exhibited a binding constant with $\mathbf{C B}[8]$ of greater than $10^{5} \mathrm{M}^{-1}$.

General procedure for irradiation experiments. In general, reaction components were sonicated for $15 \mathrm{~min}$, then reaction solutions were filtered through a Kimwipe stuffed into the end of pipette and degassed with nitrogen bubbling for $20 \mathrm{~min}$ before being irradiated for 3 days. All reactions were analyzed using a Agilent 6120 Quadrupole LC/MS.

Isolation and characterization of product $\mathbf{3} \mathbf{f}$. Product $\mathbf{3} \mathbf{f}$ was isolated by acidification of the reaction solution with a few drops of trifluoroacetic acid (TFA), followed by purification via semi-prep HPLC (water/acetonitrile $0.1 \%$ TFA). Product-containing fractions were combined and lyophilized to yield pure $\mathbf{3 f}$. The ${ }^{1} \mathrm{H}$ NMR of $\mathbf{3 f}$ is shown SX. ${ }^{1} \mathrm{H}$ NMR (500 MHz, D2O) $\delta 8.70(\mathrm{~d}, \mathrm{~J}=6.3 \mathrm{~Hz}, 4 \mathrm{H}), 7.97$ $(\mathrm{d}, \mathrm{J}=6.3 \mathrm{~Hz}, 4 \mathrm{H}), 7.91(\mathrm{~d}, \mathrm{~J}=7.9 \mathrm{~Hz}, 4 \mathrm{H}), 7.50(\mathrm{~d}, \mathrm{~J}=7.9 \mathrm{~Hz}, 4 \mathrm{H}), 4.42(\mathrm{~d}, \mathrm{~J}=9.1 \mathrm{~Hz}, 2 \mathrm{H}), 4.35$ $(\mathrm{s}, 6 \mathrm{H}), 4.10(\mathrm{~d}, \mathrm{~J}=9.2 \mathrm{~Hz}, 2 \mathrm{H}) .{ }^{13} \mathrm{C}\left\{{ }^{1} \mathrm{H}\right\}$ NMR $\left(126 \mathrm{MHz}, \mathrm{D}_{2} \mathrm{O}\right) \delta 162.46,144.89,129.48,129.45$, 127.18, 126.13, 124.62, 117.41, 115.10, 51.31, 48.27, 47.20. HRMS (ESI-ToF): $\mathrm{m} / \mathrm{z}=+479.1974$ (calc'd for [M-H]: +479.1965). Important NOE contacts are provided in Table SX. 
Determination of side products. The reaction between $\mathbf{1}$ and $\mathbf{3} \mathbf{f}$ was set up as usual (section SX) on a $10 \mathrm{~mL}$ scale at a $1.5 \mathrm{mM}$ concentration without $\mathbf{C B}$ [8]. After 10 days of $400 \mathrm{~nm}$ irradiation, the crude reaction mixture was subjected to semi-prep HPLC (water/acetonitrile, $0.1 \% \mathrm{TFA}$ ) to isolate the various products. In most cases, an insufficient quantity of material was obtained for ${ }^{13} \mathrm{C} \mathrm{NMR}$, so these assignments should not be considered as robust, synthetically valuable syntheses. However, we are confident in our use of these experiments as aids to mechanistic hypothesis.

4-formylbenzoic acid (4): ${ }^{1} \mathrm{H}$ NMR $\left(500 \mathrm{MHz}, \mathrm{D}_{2} \mathrm{O}\right) \delta 10.02(\mathrm{~s}, 1 \mathrm{H}), 8.05-8.00(\mathrm{~m}, 4 \mathrm{H}) .13 \mathrm{C}$

NMR (126 MHz, D2O) $\delta$ 214.96, 163.31, 129.59, 129.10, 116.92, 114.61. LRMS (ESI): $m / z=-149.1$ (calc'd for [M-H]: -149.0)

3g: ${ }^{1} \mathrm{H}$ NMR of major isomer $\left(500 \mathrm{MHz}, \mathrm{D}_{2} \mathrm{O}\right) \delta 7.58(\mathrm{~d}, J=8.3 \mathrm{~Hz}, 8 \mathrm{H}), 7.25(\mathrm{~d}, J=8.3 \mathrm{~Hz}, 8 \mathrm{H})$, $3.94(\mathrm{p}, J=6.2 \mathrm{~Hz}, 8 \mathrm{H})$. LRMS (ESI): $m / z=-535.1$ (calc'd for $[\mathrm{M}-\mathrm{H}]:-535.1$ )

3h: LRMS (ESI): $m / z=508.2$ (calc'd for [M]: 508.2). ${ }^{1} \mathrm{H}$ NMR of this side product was very complicated due to residual impurities and multiple isomers. However, the presence of a complicated set of overlapping doublets is consistent with the assigned structure.

Yield determination by LCMS: After completion of the reaction, a $100 \mu \mathrm{L}$ aliquot was diluted with $900 \mu \mathrm{L}$ of a $1.0 \mathrm{mg} / \mathrm{mL}$ solution of the internal standard 2,6-ditertbutyl-4-methylphenol in 9:1 acetonitrile/water with $0.1 \%$ formic acid. The resulting solution was subjected to LCMS and the relative peak areas of $\mathbf{3 f}, \mathbf{4}$, and the internal standard on the $254 \mathrm{~nm}$ chromatogram trace were determined. To convert these values to yields, we constructed a calibration curve for 4 . Lacking adequate quantities of $\mathbf{3} \mathbf{f}$ for a calibration curve, we instead subjected a sample prepared as above to ${ }^{1} \mathrm{H}$ NMR to determine the molar ratio of $\mathbf{3} \mathbf{f}$ and the internal standard. This then provided a conversion factor between LCMS peak ratios and molar ratios.

Transient Absorption. The setup for transient absorption (TA) measurements is described elsewhere. ${ }^{67}$ For these experiments, the pump was tuned to the desired wavelength for each sample (see Table Sx) with an OPA and focused onto the sample. The probe beam was generated using an $800 \mathrm{~nm}$ (100 fs) pulse routed through a delay stage and a 3-mm sapphire window to monitor the visible spectral region. The probe wavelength for kinetic analysis was taken from the peak of the excited state absorption. All kinetic traces were fit with mono-exponential decay functions convoluted with an instrument response function of 300 fs. See Table SX for time constants and wavelengths used for fitting.

\section{COMPUTATIONAL METHODS}

Computational modeling. All calculations were carried out with the ORCA 4.2.1 program. . $^{589}$ Avogadro was used to visualize orbitals. ${ }^{60}$ Ternary complexes were modeled using a B3LYP functional, 6-31G(d) basis set, and a CPCM solvent model in water. ${ }^{61,62}$ The ternary complexes are shown in Figure SX. In all ternary complexes, the potentially reactive double bonds are nearly parallel and well within the $4.2 \AA$ distance of the Schmidt criterion for [2+2] reaction. ${ }^{12}$ Geometry optimizations for individual stilbenes were performed using a B3LYP functional, 6-31G(d) basis set, 
and a CPCM solvent model in water. ${ }^{61,63-65} \mathrm{We}$ confirmed that all of the computed stilbene structures contained no imaginary frequencies. The stilbene frontier molecular orbital energies are shown in Table SX. To predict BET rates with 1, we used Eqs. 1-4, which are reproduced here.

$$
\begin{aligned}
k_{\mathrm{BET}} & =\frac{k_{B} T}{h} e^{-\frac{\Delta G^{\ddagger}}{R T}} \\
\Delta G^{\ddagger} & =\frac{\lambda}{4}\left(1+\frac{\Delta G_{\mathrm{BET}}}{\lambda}\right)^{2} \\
\Delta G_{\mathrm{BET}} & =\Delta E_{\mathrm{ET}}+w_{p}-w_{r} \\
w & =\frac{e z_{A} z_{B}}{4 \pi \epsilon_{s} \epsilon_{0} a}
\end{aligned}
$$

where $k_{B}$ is Bolzmann's constant, $T$ is temperature, $h$ is Planck's constant, $R$ is the gas constant, $\epsilon_{0}$ is the permittivity of free space, $\epsilon_{S}$ is the dielectric constant of the solvent, $\Delta G^{\ddagger}$ is the free energy of activation, $\lambda$ is the reorganization energy, $\Delta G_{\mathrm{BET}}$ is the free energy change of the back electron transfer, $\Delta E_{\mathrm{ET}}$ is the energy of the charge separated state as measured by UV-Vis, and $w_{p}$ and $w_{r}$ are the Coloumbic work terms associated with separation of products or reactants, respectively, of charges $Z_{\mathrm{A}}$ and $Z_{\mathrm{B}}$ by distance $a$ in solvent of dielectric $\epsilon_{s} \cdot{ }^{66} \mathrm{We}$ used the DFT-calculated frontier MO energies to determine $\Delta E_{\mathrm{ET}}$. We estimated the dielectric within the $\mathrm{CB}$ [8] cavity to be 10 based on the previously estimated dielectric with cucurbit[7] uril. ${ }^{44}$ We estimated the reorganization energy to be 1.6 $\mathrm{eV}$ based on measurements of single molecule conductance through $\mathbf{C B}[8]$-viologen complexes. ${ }^{50}$ Lastly, we estimated the distance between the substrates to be $3.4 \AA$ based on the distance between substrates in the DFT-calculated structures of ternary complexes above. The structures, frontier molecular energies, CT gaps with 1 (i.e. difference between LUMO energy of 1 and HOMO energy of the donor stilbene), and predicted BET rate are shown in Table SX.

\section{ACKNOLWEDGEMENTS}

This research was primarily supported by an Army Research Office Young Investigator Program grant (\#72846-CH-YIP to J.A.K.). C.T.E. acknowledges support from a National Science Foundation Graduate Research Fellowship (grant no. DGE-1842165). C.O. acknowledges support from an Academic Year Undergraduate Research Grant from Northwestern University. S.P., M.C.H., and E.A.W. acknowledge support from the Northwestern University Materials Research Science and Engineering Center (MRSEC) (NSF DMR-1720139). This work made use of the IMSERC at Northwestern University, which has received support from the Soft and Hybrid Nanotechnology Experimental (SHyNE) Resource (NSF ECCS-1542205), the State of Illinois, and the International Institute for Nanotechnology (IIN). This research was supported in part through the computational resources and staff contributions provided for the Quest high performance computing facility at Northwestern University, which is jointly supported by the Office of the Provost, the Office for Research, and Northwestern University Information Technology. 


\section{SUPPORTING INFORMATION}

Supporting Information: ${ }^{1} \mathrm{H}$ and ${ }^{13} \mathrm{C}$ NMR spectra of newly synthesized compounds, UV-vis spectra of compounds and complexes, titration experiments, LED emission profiles, control reactions, full Marcus theory treatment

\section{References}

(1) Maddipatla, M. V. S. N.; Kaanumalle, L. S.; Natarajan, A.; Pattabiraman, M.; Ramamurthy, V. Preorientation of Olefins toward a Single Photodimer: Cucurbituril-Mediated Photodimerization of Protonated Azastilbenes in Water. Langmuir 2007, 23 (14), 7545-7554. https://doi.org/10.1021/la700803k.

(2) Rao, K. S. S. P.; Hubig, S. M.; Moorthy, J. N.; Kochi, J. K. Stereoselective Photodimerization of (E)Stilbenes in Crystalline $\gamma$-Cyclodextrin Inclusion Complexes. J. Org. Chem. 1999, 64 (22), 8098-8104. https://doi.org/10.1021/jo9903149.

(3) Doi, T.; Kashida, H.; Asanuma, H. Efficiency of [2 +2] Photodimerization of Various Stilbene Derivatives within the DNA Duplex Scaffold. Org. Biomol. Chem. 2015, 13 (15), 4430-4437. https://doi.org/10.1039/C4OB02520B.

(4) Lewis, F. D.; Bedell, A. M.; Dykstra, R. E.; Elbert, J. E.; Gould, I. R.; Farid, S. Photochemical Generation, Isomerization, and Oxygenation of Stilbene Cation Radicals. J. Am. Chem. Soc. 1990, 112 (22), 8055-8064. https://doi.org/10.1021/ja00178a031.

(5) D'Auria, M.; Racioppi, R. The Photodimerisation of Coumarin. J. Photochem. Photobiol. Chem. 2004, 163 (3), 557-559. https://doi.org/10.1016/j.jphotochem.2004.02.012.

(6) Jiang, Y.; Wang, C.; Rogers, C. R.; Kodaimati, M. S.; Weiss, E. A. Regio- and Diastereoselective Intermolecular [2+2] Cycloadditions Photocatalysed by Quantum Dots. Nat. Chem. 2019, 11 (11), 1034-1040. https://doi.org/10.1038/s41557-019-0344-4.

(7) Alonso, R.; Bach, T. A Chiral Thioxanthone as an Organocatalyst for Enantioselective [2+2] Photocycloaddition Reactions Induced by Visible Light. Angew. Chem. Int. Ed. 2014, 53 (17), 43684371. https:// doi.org/10.1002/anie.201310997.

(8) Yoon, T. P. Visible Light Photocatalysis: The Development of Photocatalytic Radical Ion Cycloadditions. ACS Catal. 2013, 3 (5), 895-902. https://doi.org/10.1021/cs400088e.

(9) Pattabiraman, M.; Natarajan, A.; Kaliappan, R.; Mague, J. T.; Ramamurthy, V. Template Directed Photodimerization of Trans-1,2-Bis(n-Pyridyl)Ethylenes and Stilbazoles in Water. Chem. Commun. 2005, 36, 4542-4544. https://doi.org/10.1039/B508458J.

(10) Ischay, M. A.; Ament, M. S.; Yoon, T. P. Crossed Intermolecular [2+2] Cycloaddition of Styrenes by Visible Light Photocatalysis. Chem. Sci. 2012, 3 (9), 2807-2811. https://doi.org/10.1039/C2SC20658G.

(11) Tong, F.; Xu, W.; Guo, T.; Lui, B. F.; Hayward, R. C.; Palffy-Muhoray, P.; Al-Kaysi, R. O.; Bardeen, C. J. Photomechanical Molecular Crystals and Nanowire Assemblies Based on the [2+2] Photodimerization of a Phenylbutadiene Derivative. J. Mater. Chem. C 2020, 8 (15), 5036-5044. https://doi.org/10.1039/C9TC06946A.

(12) Ramamurthy, V.; Sivaguru, J. Supramolecular Photochemistry as a Potential Synthetic Tool: Photocycloaddition. Chem. Rev. 2016, 116 (17), 9914-9993. https://doi.org/10.1021/acs.chemrev.6b00040.

(13) Maddipatla, M. V. S. N.; Pattabiraman, M.; Natarajan, A.; Srivastav, K.; Mague, J. T.; Ramamurthy, V. Regioselective Photodimerization of Pyridyl-Butadienes within Cucurbit[8]Uril Cavities. Org. Biomol. Chem. 2012, 10 (46), 9219-9222. https://doi.org/10.1039/C2OB26743H. 
(14) Jon, S. Y.; Ko, Y. H.; Park, S. H.; Kim, H.-J.; Kim, K. A Facile, Stereoselective [2 + 2] Photoreaction Mediated by Cucurbit[8]Uril. Chem. Commun. 2001, 19, 1938-1939.

https://doi.org/10.1039/B105153A.

(15) Nakamura, A.; Irie, H.; Hara, S.; Sugawara, M.; Yamada, S. Regiospecific [2 + 2]

Photocyclodimerization of Trans-4-Styrylpyridines Templated by Cucurbit[8]Uril. Photochem. Photobiol. Sci. 2011, 10 (9), 1496-1500. https://doi.org/10.1039/C1PP05079F.

(16) Gromov, S. P.; Vedernikov, A. I.; Kuz'mina, L. G.; Kondratuk, D. V.; Sazonov, S. K.; Strelenko, Y. A.; Alfimov, M. V.; Howard, J. A. K. Photocontrolled Molecular Assembler Based on Cucurbit[8]Uril: [2+2]-Autophotocycloaddition of Styryl Dyes in the Solid State and in Water. Eur. J. Org. Chem. 2010, 2010 (13), 2587-2599. https://doi.org/10.1002/ejoc.200901324.

(17) Pemberton, B. C.; Barooah, N.; Srivatsava, D. K.; Sivaguru, J. Supramolecular Photocatalysis by Confinement-Photodimerization of Coumarins within Cucurbit[8]Urils. Chem. Commun. 2009, 46 (2), 225-227. https://doi.org/10.1039/B920605A.

(18) Ramamurthy, V.; Parthasarathy, A. Chemistry in Restricted Spaces: Select Photodimerizations in Cages, Cavities, and Capsules. Isr. J. Chem. 2011, 51 (7), 817-829. https://doi.org/10.1002/ijch.201100065.

(19) Pattabiraman, M.; Sivaguru, J.; Ramamurthy, V. Cucurbiturils as Reaction Containers for Photocycloaddition of Olefins. Isr. J. Chem. 2018, 58 (3-4), 264-275. https://doi.org/10.1002/ijch.201700100.

(20) Vallavoju, N.; Sivaguru, J. Supramolecular Photocatalysis: Combining Confinement and Non-Covalent Interactions to Control Light Initiated Reactions. Chem. Soc. Rev. 2014, 43 (12), 4084-4101. https://doi.org/10.1039/C3CS60471C.

(21) Moorthy, J. N.; Venkatesan, K.; Weiss, R. G. Photodimerization of Coumarins in Solid Cyclodextrin Inclusion Complexes. J. Org. Chem. 1992, 57 (12), 3292-3297. https://doi.org/10.1021/jo00038a012.

(22) Ji, J.; Wu, W.; Liang, W.; Cheng, G.; Matsushita, R.; Yan, Z.; Wei, X.; Rao, M.; Yuan, D.-Q.; Fukuhara, G.; Mori, T.; Inoue, Y.; Yang, C. An Ultimate Stereocontrol in Supramolecular Photochirogenesis: Photocyclodimerization of 2-Anthracenecarboxylate Mediated by Sulfur-Linked $\beta$-Cyclodextrin Dimers. J. Am. Chem. Soc. 2019, 141 (23), 9225-9238. https://doi.org/10.1021/jacs.9b01993.

(23) Zhang, C.-C.; Zhang, Y.-M.; Zhang, Z.-Y.; Wu, X.; Yu, Q.; Liu, Y. Photoreaction-Driven TwoDimensional Periodic Polyrotaxane-Type Supramolecular Nanoarchitecture. Chem. Commun. 2019, 55, 8138-8141. https://doi.org/10.1039/C9CC03705E.

(24) Guo, Q.-H.; Jia, M.; Liu, Z.; Qiu, Y.; Chen, H.; Shen, D.; Zhang, X.; Tu, Q.; Ryder, M. R.; Chen, H.; Li, P.; Xu, Y.; Li, P.; Chen, Z.; Shekhawat, G. S.; Dravid, V. P.; Snurr, R. Q.; Philp, D.; Sue, A. C.-H.; Farha, O. K.; Rolandi, M.; Stoddart, J. F. Single-Crystal Polycationic Polymers Obtained by SingleCrystal-to-Single-Crystal Photopolymerization. J. Am. Chem. Soc. 2020, 142 (13), 6180-6187. https://doi.org/10.1021/jacs.9b13790.

(25) Schmidt Bernhard V. K. J.; Barner-Kowollik Christopher. Dynamic Macromolecular Material Design-The Versatility of Cyclodextrin-Based Host-Guest Chemistry. Angew. Chem. Int. Ed. 2017, 56 (29), 8350-8369. https://doi.org/10.1002/anie.201612150.

(26) Kalayci, K.; Frisch, H.; Barner-Kowollik, C.; Truong, V. X. Wavelength-Dependent Stiffening of Hydrogel Matrices via Redshifted [2+2] Photocycloadditions. Adv. Funct. Mater. 2020, 30 (15), 1908171. https://doi.org/10.1002/adfm.201908171.

(27) Blasco, E.; Wegener, M.; Barner-Kowollik, C. Photochemically Driven Polymeric Network Formation: Synthesis and Applications. Adv. Mater. 2017, 29 (15), 1604005. https://doi.org/10.1002/adma.201604005.

(28) Horiuchi, S.; Nishioka, Y.; Murase, T.; Fujita, M. Both [2+2] and [2+4] Additions of Inert Aromatics via Identical Ternary Host-Guest Complexes. Chem. Commun. 2010, 46 (20), 3460-3462. https://doi.org/10.1039/C003191G.

(29) Nishioka, Y.; Yamaguchi, T.; Yoshizawa, M.; Fujita, M. Unusual [2+4] and [2+2] Cycloadditions of Arenes in the Confined Cavity of Self-Assembled Cages. J. Am. Chem. Soc. 2007, 129 (22), 7000-7001. https://doi.org/10.1021/ja071591x. 
(30) Yoshizawa, M.; Klosterman, J. K.; Fujita, M. Functional Molecular Flasks: New Properties and Reactions within Discrete, Self-Assembled Hosts. Angew. Chem. Int. Ed. 2009, 48 (19), 3418-3438. https://doi.org/10.1002/anie.200805340.

(31) Yoshizawa, M.; Takeyama, Y.; Okano, T.; Fujita, M. Cavity-Directed Synthesis within a SelfAssembled Coordination Cage: Highly Selective [2+2] Cross-Photodimerization of Olefins. J. Am. Chem. Soc. 2003, 125 (11), 3243-3247. https://doi.org/10.1021/ja020718+.

Clements, A. R.; Pattabiraman, M. $\gamma$-Cyclodextrin Mediated Photo-Heterodimerization between Cinnamic Acids and Coumarins. J. Photochem. Photobiol. Chem. 2015, 297, 1-7. https://doi.org/10.1016/j.jphotochem.2014.10.001.

(33) Lee, J. W.; Kim, K.; Choi, S.; Ko, Y. H.; Sakamoto, S.; Yamaguchi, K.; Kim, K. Unprecedented HostInduced Intramolecular Charge-Transfer Complex Formation. Chem. Commun. 2002, 22, 2692-2693. https://doi.org/10.1039/B208280B.

(34) Biedermann, F.; Scherman, O. A. Cucurbit[8]Uril Mediated Donor-Acceptor Ternary Complexes: A Model System for Studying Charge-Transfer Interactions. J. Phys. Chem. B 2012, 116 (9), 2842-2849. https://doi.org/10.1021/jp2110067.

(35) Lewis, T. N.; Tonnelé, C.; Shuler, W. G.; Kasun, Z. A.; Sato, H.; Berges, A. J.; Rodriguez, J. R.; Krische, M. J.; Casanova, D.; Bardeen, C. J. Chemical Tuning of Exciton versus Charge-Transfer Excited States in Conformationally Restricted Arylene Cages. J. Am. Chem. Soc. 2021. https://doi.org/10.1021/jacs.1c08176.

(36) Crisenza, G. E. M.; Mazzarella, D.; Melchiorre, P. Synthetic Methods Driven by the Photoactivity of Electron Donor-Acceptor Complexes. J. Am. Chem. Soc. 2020, 142 (12), 5461-5476. https://doi.org/10.1021/jacs.0c01416.

(37) Haga, N.; Nakajima, H.; Takayanagi, H.; Tokumaru, K. Exclusive Production of a Cycloadduct from Selective Excitation of the Charge-Transfer Complex between Acenaphthylene and Tetracyanoethylene in the Crystalline State in Contrast to Failure of Reaction in Solution. Chem. Commun. 1997, 13, 1171-1172. https://doi.org/10.1039/A702564E.

(38) Haga, N.; Nakajima, H.; Takayanagi, H.; Tokumaru, K. Photoinduced Electron Transfer between Acenaphthylene and Tetracyanoethylene: Effect of Irradiation Mode on Reactivity of the ChargeTransfer Complex and the Resulted Radical Ion Pair in Solution and Crystalline State. J. Org. Chem. 1998, 63 (16), 5372-5384. https://doi.org/10.1021/jo9801824.

(39) Haga, N.; Takayanagi, H.; Tokumaru, K. The Factor Which Determines Whether Excitation of Charge-Transfer Complexes Leads to Final Net Products in Comparison with the Reactivity on Excitation of One of the Components. Photochem. Photobiol. Sci. 2003, 2 (11), 1215-1219. https://doi.org/10.1039/B305196J.

(40) Haga, N.; Takayanagi, H.; Tokumaru, K. Photoinduced Electron Transfer between Acenaphthylene and 1,4-Benzoquinones. Formation of Dimers of Acenaphthylene and $1: 1$-Adducts and Effect of Excitation Mode on Reactivity of the Charge-Transfer Complexes. J. Chem. Soc. Perkin Trans. 2 2002, 4, 734-745. https:// doi.org/10.1039/B200098A.

(41) Ushakov, E. N.; Nadtochenko, V. A.; Gromov, S. P.; Vedernikov, A. I.; Lobova, N. A.; Alfimov, M. V.; Gostev, F. E.; Petrukhin, A. N.; Sarkisov, O. M. Ultrafast Excited State Dynamics of the Bi- and Termolecular Stilbene-Viologen Charge-Transfer Complexes Assembled via Host-Guest Interactions. Chem. Phys. 2004, 298 (1), 251-261. https://doi.org/10.1016/j.chemphys.2003.12.002.

(42) Ushakov, E. N.; Martyanov, T. P.; Vedernikov, A. I.; Sazonov, S. K.; Strelnikov, I. G.; Klimenko, L. S.; Alfimov, M. V.; Gromov, S. P. Stereospecific [2+2]-Cross-Photocycloaddition in a Supramolecular Donor-Acceptor Complex. Tetrahedron Lett. 2019, 60 (2), 150-153. https://doi.org/10.1016/j.tetlet.2018.11.077.

(43) Martyanov, T. P.; Ushakov, E. N.; Nuriev, V. N.; Aleksandrova, N. A.; Sazonov, S. K.; Vedernikov, A. I.; Kuz'mina, L. G.; Klimenko, L. S.; Martyanova, E. G.; Gromov, S. P. Pseudodimeric Complexes of an (18-Crown-6)Stilbene with Styryl Dyes Containing an Ammonioalkyl Group: Synthesis, Structure, and Stereospecific [2 + 2] Cross-Photocycloaddition. J. Org. Chem. 2021, 86 (4), 3164-3175. https://doi.org/10.1021/acs.joc.0c02514. 
(44) Masson, E.; Ling, X.; Joseph, R.; Kyeremeh-Mensah, L.; Lu, X. Cucurbituril Chemistry: A Tale of Supramolecular Success. RSC Adv. 2012, 2 (4), 1213-1247. https://doi.org/10.1039/C1RA00768H.

(60) Hanwell, M. D.; Curtis, D. E.; Lonie, D. C.; Vandermeersch, T.; Zurek, E.; Hutchison, G. R. Avogadro: An Advanced Semantic Chemical Editor, Visualization, and Analysis Platform. J. Cheminformatics 2012, 4 (1), 17. https://doi.org/10.1186/1758-2946-4-17.

(61) Becke, A. D. Density-functional Thermochemistry. III. The Role of Exact Exchange. J. Chem. Phys. 1993, 98 (7), 5648-5652. https://doi.org/10.1063/1.464913. 
(62) Stephens, P. J.; Devlin, F. J.; Chabalowski, C. F.; Frisch, M. J. Ab Initio Calculation of Vibrational Absorption and Circular Dichroism Spectra Using Density Functional Force Fields. J. Phys. Chem. 1994, 98 (45), 11623-11627. https://doi.org/10.1021/j100096a001.

(63) Becke, A. D. Density-Functional Exchange-Energy Approximation with Correct Asymptotic Behavior. Phys. Rev. A 1988, 38 (6), 3098-3100. https://doi.org/10.1103/PhysRevA.38.3098.

(64) Hehre, W. J.; Ditchfield, R.; Pople, J. A. Self—Consistent Molecular Orbital Methods. XII. Further Extensions of Gaussian-Type Basis Sets for Use in Molecular Orbital Studies of Organic Molecules. J. Chem. Phys. 1972, 56 (5), 2257-2261. https://doi.org/10.1063/1.1677527.

(65) Barone, V.; Cossi, M. Quantum Calculation of Molecular Energies and Energy Gradients in Solution by a Conductor Solvent Model. J. Phys. Chem. A 1998, 102 (11), 1995-2001. https://doi.org/10.1021/jp9716997.

(66) Wirz, J.; Klàn, P. Photochemistry of Organic Compounds, 1st ed.; John Wiley \& Sons, Ltd, 2009. https://doi.org/10.1002/9781444300017.

(67) Padgaonkar, S.; Amsterdam, S. H.; Bergeron, H.; Su, K.; Marks, T. J.; Hersam, M. C.; Weiss, E. A. Molecular-Orientation-Dependent Interfacial Charge Transfer in Phthalocyanine/MoS2 MixedDimensional Heterojunctions. J. Phys. Chem. C 2019, 123 (21), 13337-13343.

https://doi.org/10.1021/acs.jpcc.9b04063. 